\title{
A hierarchical transcriptional network activates specific CDK inhibitors that regulate G2 to control cell size and number in Arabidopsis
}

Yuji Nomoto ${ }^{1,10}$, Hirotomo Takatsuka ${ }^{1,10}$, Kesuke Yamada $^{1}$, Toshiya Suzuki², Takamasa Suzuki ${ }^{3}$, Ying Huang ${ }^{4}$, David Latrasse ${ }^{4}$, Jing An ${ }^{4}$, Magdolna Gombos $^{5}$, Christian Breuer ${ }^{6}$, Takashi Ishida ${ }^{6,11}$, Kenichiro $\mathrm{Maeo}^{7}$, Miyu Imamura ${ }^{7}$, Takafumi Yamashino ${ }^{7}$, Keiko Sugimoto $^{6,8}$, Zoltán Magyar $^{5}$, László Bögre ${ }^{9}$, Cécile Raynaud ${ }^{4}$, Moussa Benhamed ${ }^{4}$, Masaki Ito ${ }^{1}$

${ }^{1}$ School of Biological Science and Technology, College of Science and Engineering, Kanazawa University, Kakuma-machi, Kanazawa, 920-1192 Japan

${ }^{2}$ National Institute of Genetics, 1111 Yata, Mishima, Shizuoka 411-8540, Japan

${ }^{3}$ College of Bioscience and Biotechnology, Chubu University, Kasugai, Aichi 487-8501, Japan

${ }^{4}$ Université Paris-Saclay, CNRS, INRAE, Univ Evry, Institute of Plant Sciences Paris-Saclay (IPS2), 91405, Orsay, France

${ }^{5}$ Institute of Plant Biology, Biological Research Centre, Szeged, 6726, Hungary

${ }^{6}$ RIKEN Center for Sustainable Resource Science, Yokohama 230-0045, Japan

${ }^{7}$ Graduate School of Bioagricultural Sciences, Nagoya University, Furocho, Chikusa-ku, Nagoya, 464-8601, Japan

${ }^{8}$ Department of Biological Sciences, Graduate School of Science, The University of Tokyo, 7-31 Hongo, Bunkyo-ku, Tokyo 113-0033, Japan

${ }^{9}$ Royal Holloway University of London, Department of Biological Sciences, Centre for Systems and Synthetic Biology, Egham, TW20 0EX, UK

\footnotetext{
${ }^{10}$ These authors contributed equally: Yuji Nomoto and Hirotomo Takatsuka.

${ }^{11}$ Present address: Faculty of Advanced Science and Technology, Kumamoto University, Kumamoto 860-8555, Japan.

Correspondence and requests for materials should be addressed to M.I (email: masakito@se.kanazawa-u.ac.jp)
} 


\begin{abstract}
How cell size and number are determined during organ development remains a fundamental question in cell biology. Here, we identified a GRAS family transcription factor, called SCARECROW-LIKE28 (SCL28), with a critical role in determining cell size in Arabidopsis. SCL28 is part of a transcriptional regulatory network downstream of the central MYB3Rs that regulate G2 to M phase cell cycle transition. We show that SCL28 forms a dimer with the AP2type transcription factor, AtSMOS1, which defines the specificity for promoter binding and directly activates transcription of a specific set of SIAMESE-RELATED (SMR) family genes, encoding plant-specific inhibitors of cyclin-dependent kinases and thus inhibiting cell cycle progression at G2 and promoting the onset of endoreplication. Through this dose-dependent regulation of SMR transcription, SCL28 quantitatively sets the balance between cell size and number without dramatically changing final organ size. We propose that this hierarchical transcriptional network constitutes a cell cycle regulatory mechanism that allows to adjust cell size and number to attain robust organ growth.
\end{abstract}


During organ growth and development, cell proliferation is intricately controlled in space and time. Governed by developmental programs (Roeder et al., 2010; Polyn et al., 2015; Andriankaja et al., 2012) and influenced by active plant responses to environmental conditions (Komaki and Sugimoto, 2012; Qi and Zhang, 2020), the number of cells produced during organ growth is set by the cell cycle speed during proliferation and the point when cells exit to cellular differentiation. Controlled cell proliferation requires coordinately regulated gene expression within and upon exit from the cell cycle. Generally, there are two main groups of genes showing waves of transcription during the cell cycle; G1/S-specific and G2/M-specific genes (Berckmans and De Veylder, 2009). The G1/S-specific genes facilitate initiation and progression of DNA replication and are typically regulated by the activity of E2F transcription factors (Vandepoele et al., 2005; Ószi et al., 2020). Generally, E2F dimerizes with Dimerization Partner (DP) proteins to activate or repress their target genes, depending on their association with the Retinoblastoma-Related (RBR) repressor protein (Desvoyes and Gutierrez, 2020). On the other hand, most G2/M-specific genes are positively or negatively regulated by MYB3R family of transcription factors in plants (Haga et al., 2011; Kobayashi et al., 2015). Some members of MYB3Rs are specifically expressed during $\mathrm{G} 2 / \mathrm{M}$ and act as transcriptional activators, while others act as transcriptional repressors of G2/Mspecific genes (Ito et al., 2001; Haga et al., 2007; Okumura et al., 2021). Recent studies identified the two main groups of transcription factors, E2Fs and MYB3Rs, which had been studied separately, as part of the same multi-protein complex in Arabidopsis (Kobayashi et al., 2015). This E2F-MYB3R complex is evolutionarily related to the DREAM (DP, Retinoblastoma-like, E2F, and MuvB) complex reported in Drosophila and human cells (Kobayashi et al., 2015; Ning et al., 2020; Lang et al., 2021). The metazoan DREAM complex plays a predominant role in repressing both G1/S- and G2/M-specific genes, thus promoting cell cycle exit and maintaining cellular quiescence (Sadasivam and DeCaprio, 2013; Fischer and Müller, 2017). The DREAM complex in Arabidopsis shows significant differences from metazoan complexes, which include involvement of plant-specific subunits and existence of diversified complexes with different subunit compositions (Kobayashi et al., 2015; Ning et al., 2020; Desvoyes and Gutierrez, 2020).

Transcriptional regulation during cell cycle generally constitutes multi-layered hierarchical networks, in which master regulators regulate other transcription factors, which further regulate each other or downstream genes (Lee et al., 2002; Zhu et al., 2004; Millour et al., 2011). Notably, studies in yeasts showed that cell cycle transcriptional activators that function during one stage of the cell cycle regulate transcriptional activators that function during the next stage, forming a connected regulatory network that is itself a cycle (Simon et al., 2001). However, in plants, such a hierarchical network composed of cell cycle transcription factors, E2F and MYB3R, has not yet 
been uncovered. Exploring the transcription network during cell cycle may uncover missing important factors and hidden mechanisms governing plant-specific cell cycle regulation. In accordance with our work, recently a mitosis specific GRAS family transcription factor, called SCARECROW-LIKE28 (SCL28), has been identified as being directly regulated by MYB3Rs (Goldy et al., 2021). Here we demonstrate that SCL28 acts in association with the AP2-type transcription factor, AtSMOS1, to directly activate transcription of a specific set of SMR family genes, encoding plant-specific cyclin-dependent kinase (CDK) inhibitors (Kumar et al., 2015). This regulatory network inhibits the G2 to M phase transition during the cell cycle and promotes the onset of endoreplication, an atypical cell cycle consisting of repeated DNA replication without mitosis (Komaki and Sugimoto, 2012). Our study identified a G2/M regulatory pathway that controls cell cycle length, and is likely to be important in optimizing cellular functions by setting cell size and number during organ growth.

\section{Results}

\section{Identification of a mitotic GRAS-type transcription factor}

By analyzing transcripts specific in mitotic cells, we have sience identified an Arabidopsis GRAS family transcription factor that we designated E1M (Ito et al., 1999). A recent report looking for mitotic genes in the root meristem uncovered the same gene called SCL28 (Goldy et al., 2021), the name hereafter also adopted in this study. In a synchronized culture of Arabidopsis MM2d cells, we showed that this gene exhibited G2/M-specific transcript accumulation, which closely resembles that of the mitotic cyclin CYCB1;2 (Fig. 1a). As for most G2/M-specific genes, the socalled mitosis-specific activator (MSA) element that serves as a binding motif for MYB3Rs (Ito et al., 1998; Haga et al., 2011) were repeatedly present within the proximal promoter regions of $S C L 28$ (Fig. 1b). Binding of MYB3R to the $S C L 28$ promoter is supported by our published data from chromatin immunoprecipitation (ChIP) with MYB3R3 followed by high-throughput sequencing (ChIP-Seq; Fig. 1b) (Kobayashi et al., 2015) and DNA affinity purification sequencing (DAP-Seq) data reported for MYB3R1, MYB3R4, and MYB3R5 (Supplementary Fig. 1a) (O'Malley et al., 2016). In addition, loss of MYB3R activators (myb3r1/4 double mutant) or MYB3R repressors (myb3r1/3/5 triple mutant) resulted in significant down- or up-regulation of SCL28, respectively (Fig. 1c). GUS reporter activity driven by SCL28 promoter decreased significantly in myb3r1/4 double mutant and almost diminished by elimination of MSA elements (Fig. 1d). Collectively, these observations support the idea that SCL28 is a direct target of both 
activator and repressor type MYB3Rs in Arabidopsis. Similar to the G2/M-specific CYCB1;1GFP accumulation (Ubeda-Tomás et al., 2009), we observed patchy pattern of SCL28-GFP signal from a construct driven by native SCL28 promoter in root meristem, suggesting cell cycleregulated protein accumulation (Fig. 1e). Taken together, as has been recently shown (Goldy et al., 2021), SCL28 is a mitotic gene directly regulated by MYB3Rs.

To evaluate whether MYB3Rs act as part of DREAM complex on SCL28 transcription, we searched lists of target genes bound by potential DREAM components, RBR and TESMIN/TSO1LIKE CXC 5 (TCX5), as defined by ChIP-Seq experiments reported previously (Bouyer et al., 2018; Ning et al., 2020). This examination revealed that TCX5, but not RBR, showed a significant association to SCL28 locus in vivo. However, SCL28 showed no significant change in expression in neither $r b r$ nor $t c x 5 t c x 6$ double or $e 2 f a$ e2fb e2fc triple mutants, indicating that SCL28 transcription depends exclusively on MYB3R (Supplementary Fig. 1b).

\section{SCL28 strongly affects cell size}

To analyze the biological function of SCL28, we generated transgenic plants overexpressing SCL28 under the strong RPS5A promoter (proRPS5A::SCL28). These plants, herein designated $S C L 28^{\mathrm{OE}}$, showed general growth retardation both in seedling and adult stages (Fig. 2a). Cell size in these plants was significantly enlarged in all examined organs and tissues, such as root tip, leaf mesophyll, embryo, and inflorescence stem (Fig. 2b and Supplementary Fig. 2). An increased cell size was apparent in both post-mitotic differentiated cells and proliferating cell populations in root and shoot apical meristems (Fig. 2c and Supplementary Fig. 2). As described later, the increased cell size in $S C L 28^{\mathrm{OE}}$ leaves was associated with elevated levels of cellular ploidy induced by enhanced endoreduplication. The loss-of-function scl28 mutant (Supplementary Fig. 3) showed an opposite cellular phenotype to $S C L 28^{\mathrm{OE}}$, having cells with significantly reduced size (Fig. 2b, c and Supplementary Fig. 2). However, unlike SCL28 ${ }^{\mathrm{OE}}$, the overall stature of $s c l 28$ mutant plants was largely normal and indistinguishable from that of wild type (WT) plants (Fig. 2a).

To explore the developmental origin of cell size differences, we performed kinematic analysis on growing first leaf pairs by monitoring size and number of palisade cells (Fig. 2d-g). This analysis showed that cells in scl28 leaves were smaller than WT already during initial stages of organ development when most cells are actively proliferating (Fig. 2d). During this early proliferative stage, increasing cell number was also more rapid in $s c l 28$ than WT, consistent with a higher cell division rate in scl28 leaves compared with WT (Fig. 2e, f). Conversely, duration of 
cell proliferation remained largely unchanged between $s c l 28$ and WT (Fig. 2e, f). Therefore, the increased cell number in $s c l 28$ leaves is due to accelerated cell division, rather than increased duration of cell proliferation. When leaf area was compared, however, scl28 and WT showed no clear difference throughout the course of leaf development (Fig. 2g). In scl28 leaves, accelerated cell division was balanced by reduced cell size, thus maintaining total organ size unchanged during leaf development. The cellular effect of $S C L 28^{\mathrm{OE}}$ was generally opposite to the $s c l 28$ mutant. The number of palisade cells per leaf was significantly reduced in $S C L 28^{\mathrm{OE}}$ plants (Fig. 2e) due to severely inhibited cell proliferation (Fig. 2f). Although dramatic cell enlargement partially counteracted the reduced cell number, leaf area was still reduced in SCL2 ${ }^{\circ \mathrm{OE}}$ compared with WT (Fig. 2d, g).

In the root tip, cell size difference was similarly apparent among $S C L 28^{\mathrm{OE}}, s c l 28$, and WT (Fig. 2c, h, i). This cell size difference was maintained along the apical-basal axis of the root from cells adjacent to the quiescent center to those at the transition zone, as well as in differentiated cells at distal positions (Fig. 2h). Therefore, the behavior of cells in the root meristems of SCL28 ${ }^{\mathrm{OE}}$ and scl 28 was generally consistent with those observed in developing leaves.

\section{Effects of SCL28 on mitotic cell cycle and endocycle}

To directly explore the role of SCL28 in cell cycle progression, we performed live-cell imaging of root meristem cells after introgression of the PCNA-GFP cell cycle marker into SCL28 ${ }^{O E}$ and scl28 mutant. The PCNA-GFP line allows quantification of cell cycle stages based on its intracellular fluorescent patterns (Yokoyama et al., 2016). During G1, PCNA-GFP appears as uniform fluorescence within the whole nuclei, which alters into dotty and speckled nuclear signals during early and late S phase, respectively. During G2, fluorescence again becomes uniform within the nuclei, then disappears upon onset of mitosis and remains undetectable until the exit from mitosis. By our definition, the G1 period begins with re-appearance of nuclear fluorescence after mitosis and ends with emergence of dotty GFP signals, whereas the G2 period begins with conversion of dotty into uniform nuclear PCNA-GFP signals and ends with disappearance of this nuclear fluorescence. Based on these definitions, we analyzed data from live-cell imaging and calculated the average duration of each cell cycle phase in WT root meristem cells to be $5.1 \mathrm{~h}$ in G1, $2.5 \mathrm{~h}$ in S, and $11.3 \mathrm{~h}$ in G2 (Fig. 3a, b). G2/M duration was oppositely affected in the scl28 and SCL2 $8^{\mathrm{OE}}$ lines, shortened $19 \%$ in the former and lengthened $33 \%$ in the later. In contrast, G1 length showed only limited alteration, but became longer in the scl28 mutant (Fig. 3a, b), which is consistent with the existence of an additional cell size checkpoint at G1/S that compensates for shortened G2 (Jones et al., 2017). This suggests that SCL28 actively inhibits progression through 
G2 and prevents entry into mitosis. In agreement with the role of SCL28 in G2 duration of the mitotic cell cycle, our ploidy analysis of developing leaves showed that SCL28 also affects endoreplication (Fig. 3c, d, and Supplementary Fig. 4). During leaf development, SCL28 ${ }^{\mathrm{OE}}$ plants initiated earlier endoreplication, indicated by $8 \mathrm{C}$ cell emergence as early as eight days after sowing (DAS) and thereafter consistently showing higher cellular ploidy levels compared with WT. Elevated ploidy levels were associated with dramatically increased cell size in SCL28 ${ }^{\circ}$ leaves as shown earlier (Fig. 2b, d). In the scl28 mutant, ploidy levels were not affected during early stages of leaf development and only showed a modest decrease at 20 DAS. During the earliest stage (8 DAS) before onset of endoreplication, we observed a smaller proportion of $4 \mathrm{C}$ cells in scl28 compared with WT (Fig. 3c), which supports the cell cycle analysis, showing reduced G2 duration (Fig. 3a). In summary, our data suggest that SCL28 has a role to inhibit the G2 to M phase transition.

\section{SCL28 acts together with AtSMOS1 as a heterodimer}

Phylogenetic analysis of GRAS family proteins indicated that SCL28 constitutes a unique clade together with SMOS2 in rice (Supplementary Fig. 5a). Rice smos 2 was initially identified as a mutant showing reduced organ size with component cells smaller than those in WT (Hirano et al., 2017). Another rice mutant, $\operatorname{smos} 1$, with a phenotype similar to that in $\operatorname{smos} 2$, has loss-of-function mutation in a unique gene encoding an AP2-type transcription factor (Aya et al., 2014). Because a physical interaction between SMOS1 and SOMS2 has been reported (Hirano et al., 2017), we postulated that SCL28 may act through interaction with an Arabidopsis protein orthologous to SMOS1. When AP2-type transcription factors were phylogenetically analyzed, we found At2g41710 to be the likely Arabidopsis ortholog of SMOS1 (Supplementary Fig. 5b). To test the physical interaction between SCL28 and At2g41710, we performed yeast two-hybrid and bimolecular fluorescence complementation (BiFC) assays to obtain clear results showing that they indeed interact in yeast and in plant cells, respectively (Fig. 4a, b). We concluded that the observed protein-protein interaction is evolutionarily related to the SMOS1-SMOS2 interaction in rice and named At2g41710 as AtSMOS1.

To examine AtSMOS1 function, we analyzed a T-DNA insertion mutant for this gene (Supplementary Fig. 6) and found a small cell size phenotype that closely resembled that of $s c l 28$ (Fig. 4c-f). However, the overall stature of atsmos 1 was largely indistinguishable from WT plants, as is the case for $s c l 28$ (Supplementary Fig. 7a). To analyze epistasis between $s c l 28$ and atsmos 1 , we performed genetic analysis by quantitatively comparing phenotypes of $s c l 28$, atsmos 1 and scl28 atsmos 1 double mutants. These single and double mutants showed essentially equivalent 
phenotypes in terms of palisade cell size (Fig. 4c, d) and cell size in the root meristem (Fig. 4e, f). This indicates that, as with SCL28, AtSMOS1 also impacts cell size. The lack of additive effect between atsmos 1 and $s c l 28$ suggests that these proteins may act in the same pathway, which is consistent with the interaction between these proteins. We then tested whether the enlarged cells in SCL28 ${ }^{\circ \mathrm{E}}$ relies on the presence of AtSMOS1, and indeed in the atsmos 1 mutant background, the strong effects of $S C L 28^{\mathrm{OE}}$ on cell size of leaf palisades (Fig. $4 \mathrm{~g}, \mathrm{~h}$ ) and root meristem cells (Fig. 4i, j), as well as whole plant growth, completely diminished (Supplementary Fig. 7b). Collectively, these observations strongly suggest that SCL28 and AtSMOS1 function cooperatively to regulate cell size in different plant organs.

To analyze AtSMOS1 expression, we generated plants expressing AtSMOS1-GFP driven by its own promoter. These transgenic plants showed nuclear localization of AtSMOS1-GFP in various tissues and organs such as root meristem, developing leaves, and cotyledons (Fig. 4k). However, unlike SCL28, AtSMOS1-GFP was uniformly expressed in meristematic cells, suggesting a cell cycle-independent AtSMOS1 expression. Nonetheless, cells expressing AtSMOS1 and SCL28 were overlapping, supporting the idea that they interact to form a heterodimer in vivo. We also noted an E2F binding element in the AtSMOS1 promoter, which binds RBR in published ChIP-Seq data (Bouyer et al., 2018). This highlights the possibility that, while SCL28 shows mitotic control through the MYB3Rs, AtSMOS1 may be regulated by the RBR-E2F pathway.

\section{Downstream targets of SCL28}

Studies of smos 1 and smos 2 rice mutants suggest a role in post-mitotic cell expansion through the regulation of the Oryza sativa PHOSPAHATE INDUCEDI (OSPHI-1) gene (Hirano et al., 2017). Because we observed SCL28 expression specific to meristematic cells, we postulated that SCL28 should largely influence actively-proliferating cells before the onset of cell expansion. To identify the downstream targets of SCL28, we analyzed genome-wide gene expression changes in $s c l 28$, $S C L 28^{\mathrm{OE}}$, and atsmos 1 by conducting RNA-sequencing (RNA-Seq) and microarray experiments. Considering the epistatic phenotypes among scl28, SCL28 ${ }^{\mathrm{OE}}$, and atsmos 1 mutants, the critical downstream genes of SCL28 should be affected in scl28 and atsmos 1 in a similar manner, as well as in $S C L 28^{\mathrm{O}}$ in an opposing manner. To reveal the genes satisfying these expression criteria, we conducted an overlapping analysis of differentially expressed genes in $s c l 28$, atsmos 1 , and $S C L 28^{\mathrm{OE}}$, and identified 21 genes that are significantly (adj $P$-value less than 0.05 ) downregulated in both $s c l 28$ and atsoms1, and upregulated in $S C L 28^{\mathrm{OE}}$ (Fig. 5a and Supplementary Fig. 8a), as well as 26 genes affected in an opposing way within each line (Fig. 5b and Supplementary Fig. 
8b). Among these genes, we focused on SIAMESE-RELATED2 (SMR2), encoding a member of SMR family proteins, as proteins of this family represent plant-specific CDK inhibitors and some members, such as SIM and SMR1, positively affect cell size and negatively influence cell division, reflecting the observed effect of SCL28 (Roeder et al., 2010; Kumar et al., 2015). According to RNA-Seq and microarray data, in addition to $S M R 2$, we found other $S M R$ family members as candidates of downstream genes, with decreased expression levels in both scl28 and atsmos 1 and increased levels in $S C L 28^{\mathrm{OE}}$. To further analyze $S M R$ family genes, we conducted quantitative reverse-transcription PCR (qRT-PCR) analysis to examine expression of all members of this family in $s c l 28$, atsmos 1 , and SCL2 $8^{\mathrm{O}}$, and identified at least seven members (SMR1, SMR2, SMR6, SMR8, SMR9, SMR13, and SMR14) out of all 17 as potential target genes of SCL28 and AtSMOS1 (Fig. 5c-e). Consistent with this conclusion, we also observed reduced expression of proSMR2::SMR-GFP and proSMR13::SMR13-GFP in root meristems in scl28 or atsoms 1 mutant backgrounds compared with WT (Supplementary Fig. 9).

KIP-RELATED PROTEINs (KRPs) constitute another family of CDK inhibitors in addition to SMRs in plants. The general view is that KRP proteins primarily connect with CDKA to negatively regulate G1/S (Verkest et al., 2005), while SMR proteins inhibit G2/M by associating with both CDKA and CDKB1 (Kumar et al., 2015; Wang et al., 2020). There are seven KRP members encoded in the Arabidopsis genome, none of which showed significant change in expression for all scl28, atsmos1, and SCL28 ${ }^{\mathrm{OE}}$ plants in our transcriptome analysis (Supplementary Fig. 10). Therefore, SCL28 and AtSMOS1 affect expression of SMR members, but not those of the KRP family, supporting our results that SCL28 preferentially affects G2 duration in meristematic cells.

\section{Genome-wide mapping of SCL28 and AtSMOS1 binding sites}

In order to define direct targets for SCL28 and AtSMOS1, combined with transcriptome analysis, we performed ChIP-Seq assays using proRPS5A::SCL28-GFP and proAtSMOS1::AtSMOS1GFP lines to identify their binding genomic loci. Consistent with our emphasis on downregulation of SMR genes, which suggests that SCL28 and AtSMOS1 act as transcriptional activators, the majority of binding sites are located in promoter regions proximal to transcription start sites (TSS) (Supplementary Fig. 11) and located within nucleosome-free and highly accessible chromatin regions (Supplementary Fig. 12a-d). Their enrichment levels at TSS correlated well with mRNA levels of corresponding genes, suggesting a positive correlation between their binding and transcriptional activity (Supplementary Fig. 12e, f).

The ChIP-Seq data analysis revealed 463 and 4,287 genes as targets of SCL28 and AtSMOS1, 
respectively. Comparing these target genes revealed the presence of a significant overlap, a set of 308 common targets that accounts for $66 \%$ of SCL28 targets (Fig. 6a). On one hand, this result confirms our genetic analysis, suggesting that SCL28 function largely relies on the interaction with AtSMOS1. On the other hand, AtSMOS1 may show a wider range of functions both dependent on and independent of SCL28. We found only limited overlap between common targets identified by ChIP-Seq and those that show regulated expression in transcriptome analysis of scl28, atsmos 1 and SCL28 ${ }^{\mathrm{OE}}$ lines (Fig. 6b). Gene ontology (GO) enrichment analysis of the 308 common targets revealed overrepresented GO terms related to cell cycle, such as "regulation of mitotic nuclear division" and "regulation of DNA endoreduplication" (Supplementary Fig. 13). This overrepresentation of cell cycle-related GO terms largely relies on the presence of five common genes that belong to such GO categories. All these genes were found to be members of $S M R$ family genes, SMR2, SMR4, SMR6, SMR8, and SMR9, all of which, except SMR4, showed significant downregulation in both scl28 and atsmos 1 and upregulation in SCL28 ${ }^{\text {OE }}$ in our qRTPCR experiments described above (see Fig. 5). Though three SMR genes-SMR1, SMR13, and SMR14 - with significant expression changes in all lines failed to fulfill the criteria of ChIP-Seq data analysis, visual inspection revealed recognizable peaks for both SCL28 and AtSMOS1 in ChIP-Seq profiles, suggesting these SMRs are also direct common targets (Fig. 6c and Supplementary Fig. 14). The ChIP-Seq peaks were observed at either 5' (SMR1, SMR2, and SMR4), 3' (SMR6, SMR9, and SMR14) or both 5' and 3' (SMR8 and SMR13) regions of the target $S M R$ loci. In most cases, we found that the positions of ChIP-Seq peaks adjacent to SMR loci coincided for SCL28 and AtSMOS1, suggesting that they associate with the same sites.

To find common binding sites and sequence motifs for SCL28 and AtSMOS1, we compared the precise positions of ChIP-Seq peaks along the gene structure. Dot-plot analysis of the common targets showed frequent clustering of SCL28 and AtSMOS1 peaks at the same positions relative to TSS and transcription end site (TES; Fig. 6d). Similar analysis at a genome-wide scale confirmed that SCL28 frequently targets the same sites as AtSMOS1 around the TSSs (Supplementary Fig. 15a). Within the common peaks for SCL28 and AtSMOS1, a DNA motif of $\mathrm{C}(\mathrm{a} / \mathrm{t}) \mathrm{T}(\mathrm{a} / \mathrm{t})$ GGATNC$(\mathrm{c} / \mathrm{t})(\mathrm{a} / \mathrm{t})$ could be identified as an overrepresented cis element. This motif is indeed present among more than $40 \%$ of the common targets, but is much less frequent among targets of either SCL28 or AtSMOS1, which contain quite different cis elements based on sequence overrepresentation analysis (Supplementary Fig. 15b, c). This suggests that the formation of SCL28-AtSMOS1 heterodimer generates a specific sequence preference for DNA binding to regulate transcription of a specific set of targets. This or closely related motifs are present in all seven SMR target loci at positions frequently coinciding with the peak summits of 
SCL28 and AtSMOS1 (Fig. 6c and Supplementary Fig. 14), suggesting that SCL28 and AtSMOS1 recognize this motif and bind to their targets as a heterodimer.

We then tested whether chromatin association of SCL28 depended on AtSMOS1 and viceversa by conducting ChIP-qPCR experiments in scl28 and atsmosl backgrounds (Fig. 6f). As expected, binding of both SCL28 on SMR2 promoter in the atsmos1 and AtSMOS1 binding in the scl28 mutant backgrounds were abolished. Collectively, our data supports SCL28 and AtSMOS1 functional heterodimer binding to specific sets of SMR target genes to activate transcription and negatively regulate cell cycle progression at the G2 to M cell cycle transition.

\section{SMRs are critical downstream effectors for SCL28 and AtSMOS1}

If SMR genes directly bound by SCL28 and AtSMOS1 are crucial for mediating their effects on cellular phenotype, transcript levels of those SMR genes should be altered accordingly with cell size phenotype observed in our epistasis analysis (see Fig. 4). Confirming this idea, downregulation of target $S M R$ genes - SMR2, SMR9 and SMR13 - were quantitatively equivalent in scl28, atsmos1, and double scl28 atsmos1 mutants (Fig. 7a). In addition, strong activating effects of $S C L 28^{\mathrm{OE}}$ on target transcription was totally abolished under atsmos 1 mutant background, providing the molecular basis for AtSMOS1-dependent action of SCL28 on cell size (Fig. 7b). To further elucidate that AtSMOS1 acts together with SCL28 on SMR genes, we also analyzed the proSMR2::LUC reporter co-transfected in protoplasts with pro35S::SCL28 and/or pro35S::AtSMOS1 constructs. The most prominent and significant activation of luciferase was only observed when both plasmids expressing SCL28 and AtSMOS1 were simultaneously transfected, supporting the conclusion that SCL28 and AtSMOS1 fulfill their function as a transcriptional activator by forming a heterodimer (Fig. 7c).

To directly address whether these SMR genes are critical for SCL28 function, we performed genetic analysis focusing on $S M R 1, S M R 2$ and $S M R 13$, which are significantly downregulated in $s c l 28$ and upregulated in SCL2 ${ }^{\mathrm{O}}$. For each $s m r$ mutant, we could not detect any abnormalities in cell size (Supplementary Fig. 16). However, when these mutations were combined in a smr1/2/13 triple mutant, there was a significant reduction in cell size in multiple tissues, including leaf palisades (Fig. 7d, e) and root meristem (Fig. 7f, g), suggesting functional redundancy among SMR genes. To address the link between SCL28 and SMRs, we studied the cell size phenotype when these mutations were combined. In leaf palisade cells, the triple smr1/2/13 mutant showed stronger reduction in cell size than the scl28 mutant, which did not further reduce when all mutations combined in the smr1/2/13 scl28 line, supporting the idea that cell size regulation by SCL28 is mediated by SMR1/2/13 (Fig. 7d, e). Conversely, scl28 mutation still significantly 
decreased cell size in the root meristem when combined with smr1/2/13 (Fig. 7f, g). Therefore, SMRI/2/13 contribution downstream of SCL28 appears to be larger in palisade tissue than root meristem, where additional SCL28-regulated SMRs may play a role in cell size control. These tissue-specific differences indicate the contribution of developmental regulation that positions different sets of SMRs downstream of SCL28.

\section{Dose-dependent control of cell size and cell number by SCL28 to maintain organ size homeostasis}

In the $s c l 28$ mutant, leaf growth is essentially normal, but the constituent cells became small and more numerous (Fig. 2d, e, g), suggesting that SCL28 is pivotal to maintain organ size homeostasis by regulating the balance between cell size and cell number. For a regulator designed to tune the balance between cellular parameters, one assumes that it acts dose dependently. To explore whether or not SCL28 levels quantitatively affect cell size, we analyzed plants heterozygous for $s c l 28$ ( $s c l 28 /+$ plants). Heterozygous plants showed reduced SCL28 transcript levels that is approximately half of that in WT (Fig. 8a). This SCL28 downregulation resulted in a clear reduction of cell size in root meristem and leaf palisade tissue, with an intermediate cell size in heterozygous plants between those in WT and scl 28 homozygous plants (Fig. 8b, c and Supplementary Fig. 17a, b).

Overexpression of $S C L 28$ causes a phenotype opposite to $s c l 28$, dramatically reducing the number of cells that became enlarged. To examine the outcome when SCL28 expression is only moderately increased, we utilized plants from different T2 lines carrying proSCL28::SCL28-GFP in scl28 mutant background and showing varying levels of SCL28-GFP expression. We quantitatively compared levels of SCL28 transcript and cellular parameters of palisade tissues in each individual plant (Fig. 8d, e and Supplementary Fig. 17c). This showed that SCL28 expression positively correlates with cell size and negatively with leaf cell number. Both positive and negative correlations were statistically significant. Due to compensatory changes in cell number and size, overall leaf size was not dramatically affected by moderate alteration of SCL28 expression (Fig. 8f). Therefore, we concluded that a finely-graded expression of SCL28 sets the balance between cell size and number without altering organ growth as a whole. This mechanism may enable plants to achieve an optimal balance between cell size and number, possibly depending on developmental status and environmental conditions.

\section{Discussion}


Cell size at division depends on coordination between cell growth and cell cycle progression, which is actively maintained in a cell autonomous manner in both uni- and multicellular organisms (Zatulovskiy and Skotheim, 2020; Jones et al., 2019; D'Ario and Sablowski, 2019). Here, we identified a molecular mechanism for cell size regulation in Arabidopsis that relies on a hierarchical transcriptional activation of CDK inhibitors. In this pathway, SCL28 expression is specifically confined to mitosis through the MSA element in its promoter controlled by MYB3Rs. In turn, SCL28 associates with AtSMOS1, a transcription factor with cell cycle-independent expression, and this dimer defines the binding site present in a set of SMR genes encoding CDK inhibitors to activate their expression and thereby act as a brake against the cell cycle engine predominantly driven by CDK activity (Fig. 9). The SCL28-AtSMOS1-SMR axis uniquely affects only cell cycle progression and cell doubling time, but not the exit from cell proliferation. Therefore, it acts to set the balance between cell number and size during organ development without significantly impacting final organ size. Whether SCL28 is part of a cell size sensor and how it is coupled to the measurement of cell growth remain to be determined.

We provide multiple lines of evidence supporting the conclusion that SCL28 acts as a brake on the G2/M transition to set cell size: 1) measurements of cell cycle phases by following PCNAGFP using live-cell imaging showed that $s c l 28$ mutant and $S C L 28^{\mathrm{OE}}$ shortened or lengthened the G2 phase, respectively; 2) kinematic analysis of cellular parameters during leaf development of scl 28 and SCL $28^{\mathrm{OE}}$ showed a clear alteration in cell doubling time without any effect on cell cycle exit; 3) the molecular mechanisms identified here are fully consistent with the cell cycle inhibitory function, SCL28, forming a dimer with AtSMOS1 and directly activating the transcription of SMR genes whose products inhibit CDK activity; 4) in support of this, the scl28 mutant phenocopied and interacted in an epistatic fashion with the triple smr 1/2/13 mutant; and 5) SCL28 regulates the number of endoreplication cycles, which is typically enhanced by G2 inhibition. Previous reports suggest that SCL28 plays a positive role in root growth and promotes progression through the G2/M cell cycle progression (Choe et al., 2017; Goldy et al., 2021). These conclusions are inconsistent with our data and the small cell size phenotype observed in both studies, which may originate from using different methods relying on fixed samples.

Another example of a transcription factor inhibiting cell cycle at $\mathrm{G} 2 / \mathrm{M}$ is a NAC-type transcription factor, SUPPRESSOR OF GAMMA RESPONSE 1 (SOG1), acting as a central regulator of DNA damage response in Arabidopsis. Similar to SCL28, SOG1 transcriptionally activates $S M R$ family genes to inhibit cell cycle progression upon DNA damage (Yi et al., 2014), but its targets, SMR5 and SMR7, are not regulated by SCL28, which instead directly regulates 
seven other SMR genes. Notably, in contrast with SOG1, which is activated specifically upon DNA damage, SCL28 negatively regulates cell cycle, thereby affecting cell size during normal plant development. The target of rapamycin (TOR) signaling pathway is a primary regulator of cell growth and proliferation and is involved in cell size regulation in yeast (Davie and Petersen, 2012). In Arabidopsis, TOR, acting through YAK1 kinase, regulates the transcription of SMR genes and thereby G2/M transition (Barrada et al., 2019). However, the set of YAK1-targeted SMRs overlap with those regulated by SOG1 rather than those regulated by SCL28. Therefore, our study places SCL28 as part of a novel pathway for regulating G2/M progression that determines cell size and cell number during plant organ development.

Variability in cell size at birth or stochastic changes in key regulators necessitate mechanisms that achieve cell size homeostasis by linking cell cycle progression to cell growth (D'Ario and Sablowski, 2019). The way cell size variations are corrected has long been debated. The principal possibilities are using (i) a cell cycle timer, (ii) cell size measurements or (iii) correcting cell size by adding a constant increase to daughters regardless of initial sizes (Zatulovskiy and Skotheim, 2020). Comparing predicted outcomes of these theoretical models with time-lapse imaging data on cell size in plant meristematic cells identifies possible co-existence of a mix of these mechanisms that may act at multiple control points of the cell cycle (Willis et al., 2016; Jones et al., 2017). The molecular device for cell size control may rely on cell size-dependent accumulation of an activator or dilution of a cell cycle inhibitor molecule. The 'inhibitor dilution model' postulates that cell growth dilutes such inhibitor protein until its concentration meets a threshold, thereby permitting cell division when cells reach an appropriate size (Fantes et al., 1975). This hypothesis has been verified in yeast and mammalian systems (Schmoller et al., 2015; Zatulovskiy et al, 2020). In Arabidopsis, recent studies show segregation of set amounts of chromatin-bound KRP4 CDK inhibitors act as a cell size sensor that is diluted by cell growth and is responsible for cell size-dependent cell cycle regulation at G1/S (D'Ario et al., 2021).

Live-cell imaging of cell cycle markers in Arabidopsis shoot apical meristem demonstrated that both $\mathrm{G} 1 / \mathrm{S}$ and $\mathrm{G} 2 / \mathrm{M}$ transitions are regulated in a cell size-dependent manner. Accumulation of two waves of CDK activities underpinned by D-type cyclin and mitotic CDKB1, their interactors or downstream effectors were proposed as molecular components for cell sizedependent G1/S and G2/M regulation, respectively (Jones et al., 2017). SCL28 may participate in regulating G2/M transition in this model, in which dilution of an inhibitor rather than accumulation of an activator is the principle behind. How SCL28 is connected to monitoring of cell size remains to be determined. Stochastic variability in the accumulation of key regulators can also set cell size and cell fate differences in a developing organ. An example is ATML1, a 
transcription factor that is necessary for the patterning of giant cells within the sepal epidermis by overcoming a threshold in G2 cells and activating the downstream SMR1, also called LOSS OF GIANT CELLS FROM ORGANS (LGO) (Meyer et al., 2017). Similar to ATML1, SCL28 shows a finely-tuned dose-dependent effect on cell size by accurately distinguishing gene copy numbers of SCL28 and reflecting its graded overexpression. It is possible that the molecular ruler in an inhibitor dilution model potentially involving SCL28 is set by the synthesis rate limited by gene copy number, as shown for Whi5 in budding yeast (Schmoller et al., 2015).

The human functional orthologue of Whi5 is pRB, which also controls cell size via its synthesis mostly after G1 and subsequent dilution during growth (Zatulovskiy et al, 2020). In Arabidopsis, E2FB can strongly affect cell size in proliferating cells (Magyar et al., 2005), but as opposed to SCL28 that solely acts on cell proliferation rate, the RBR-E2F pathway also regulates cell cycle exit, establishment of quiescence, and cellular differentiation (Borghi et al., 2010; Bouyer et al., 2018, Dewitte et al., 2003). The repressor-type MYB3Rs-MYB3R1, MYB3R3 and MYB3R5 - when combined with RBR and E2FC form the DREAM complex that represses mitotic genes during or after developmental cessation of cell proliferation (Kobayashi et al., 2015). It appears that SCL28 acts independently of DREAM regulatory mechanisms, which explains why cell cycle exit and concomitant cell differentiation remain unaffected, but it controls cell cycle length specifically during proliferation.

In addition to cell size during proliferation, SCL28 also strongly affects the size of terminally differentiated cells. In the $s c l 28$ mutant, we observed a reduced final cell size in various organs, whereas moderately higher SCL28 expression led to its increase. Reduced cell size caused by increased cell division, as observed in $s c l 28$, has been repeatedly reported in Arabidopsis organ development. Examples include plants with inhibited $R B R$ expression, those overexpressing CYCD3; 1, or co-overexpressing E2FA and DPA (Dewitte et al., 2003; Borghi et al., 2010; Gutzat et al., 2011; De Veylder et al., 2002). In many of these cases, the cellular phenotype is due to prolonged cell proliferation and delayed cell differentiation, leading to additional cell division that is not balanced by cell size increase due to developmental decrease of cell expansion. On the contrary, we showed with kinematic analysis during leaf development that the increased number of smaller cells observed in scl28 leaves is caused by accelerated cell proliferation, but not by prolonged cell proliferation. Therefore, it is puzzling how loss of SCL28 affects final cell size that is largely determined by post-mitotic cell expansion occurring in the absence of SCL28.

Our observations are reminiscent of the phenomena reported in Drosophila wings, where the alteration of cell number is compensated by changes in cell size such that final organ size is essentially unchanged. Based on this and similar observations, it has been proposed that animal 
organ size may be controlled by 'total mass checkpoint' mechanisms, which operate at the level of whole organs (Potter and $\mathrm{Xu}, 2001$ ), although the underlying molecular details remain unknown. Instead, the well-established mechanism of 'compensation' in plants shows postmitotic cell expansion that is enhanced by reduced cell proliferation (Hisanaga et al., 2015). A small number of larger cells observed in $S C L 28^{\circ}$ leaves may be a prime example of compensation. One possible explanation for compensatory alteration of cell expansion is that, during cell proliferation, cells establish memory that is maintained during the elongation phase. An example of this can be seen in plants overexpressing $K R P 2$, where final cell size may be influenced by the size of mitotic cells that are already altered during proliferation (Ferjani et al., 2007). We observed larger and smaller mitotic cells both in root meristem and young leaves of $S C L 28^{\mathrm{OE}}$ and $s l c 28$, respectively, which may be maintained until terminal differentiation, thus affecting final cell size. A possible molecular mechanism to create such memory is cell sizedependent alteration in gene expression. It was shown in yeast that cell size changes due to ploidy levels are associated with altered gene expression related to functions of cell wall and extracellular matrix (Wu et al., 2010). Similarly, in Arabidopsis root, ploidy levels were correlated with expression of genes related to chromatin and cell expansion, such as ion transport and cell wall modifications (Bhosale et al., 2018). Therefore, it is plausible that SCL28 action in meristematic cells has long-lasting effects through cell expansion on final cell size in differentiated tissues. Another possible mechanism for cell size memory involves a monitoring system for cellular dimensions that shares components in proliferating and expanding cells. Supporting this idea, it has been suggested that cells expand to a target size by a mechanism requiring a cell size measurement device, from an approach combining quantitative analysis and mathematical modeling of variability in final cell size of Arabidopsis root (Pavelescu et al., 2018). Further careful studies are required to clarify how final cell size is affected by expression levels of SLC28 that is confined to proliferating cells.

In summary, we identified a novel hierarchical MYB3R-SCL28/AtSMOS1-SMR transcriptional regulatory pathway leading to cell cycle inhibition at $\mathrm{G} 2 / \mathrm{M}$ that regulates cell size and number during organ development without dramatically altering organ size. This mechanism may help to adjust cell size to optimize cellular and tissue performance such as metabolic functions or mechanical properties.

\section{Methods}




\section{Plant materials}

Arabidopsis thaliana Columbia (Col) was used as the WT plant. All mutants and transgenic lines used in this study were in a Col background. The mutant alleles scl28-1 (SALK_205284), atsmos1-1 (SALK_111105C), smr1-1 (SALK_033905), smr2-1 (SALK_006098C), and smr13-1 (SALK_053256C) were identified from SALK T-DNA collection, and used for phenotype analysis and generating multiple mutant combinations. Other mutants and transgenic lines, namely myb3r1-1, myb3r3-1, myb3r4-1, myb3r5-1, PCNA-RFP, and CYCB1;1-GFP, were described previously (Haga et al, 2007; Kobayashi et al., 2015; Yokoyama et al., 2016; UbedaTomás et al., 2009). Sterilized seeds were germinated on one-half-strength Murashige and Skoog $(1 / 2 \mathrm{MS})$ medium containing $2 \%(\mathrm{w} / \mathrm{v})$ sucrose and $0.6 \%(\mathrm{w} / \mathrm{v})$ agar. Plants were grown on $1 / 2$ MS agar medium or soil under continuous light at $22^{\circ} \mathrm{C}$. For root phenotype analysis, plants were gown on a vertical surface of 1/2 MS medium containing $1.0 \%(w / v)$ agar.

\section{Gene expression analysis}

For comparing transcriptome of WT and $S C L 28^{\circ}{ }^{\mathrm{OE}}$, microarray analyses were performed using an ATH1 GeneChip ${ }^{\circledR}$ (Affymetrix) as described previously (Kobayashi et al., 2015). For RNA-Seq analyses, cDNA libraries were constructed with the TruSeq RNA Library Preparation Kit v2 (Illumina, United States) and sequenced using the NextSeq500 sequencer (Illumina) as described previously (Okumura et al., 2021). RNA-Seq data for WT, $s c l 28$, and atsmos 1 can be accessed from the DDBJ database under accession number DRA012786. RNA extraction and qRT-PCR were performed as described previously (Okumura et al., 2021), using primers listed in Supplementary Table 1.

\section{Yeast two-hybrid assay}

Yeast two-hybrid assays were performed as described by Soyano et al. (2003). Saccharomyces cerevisiae strain L40 was transformed with pBTM116- and pVP16-based plasmids carrying coding sequences encoding for SCL28 or AtSMOS1. A single colony was diluted with water and spotted on medium lacking His supplemented with $5 \mathrm{mM} 3-\mathrm{AT}$ and cultured at $30^{\circ} \mathrm{C}$ for two days.

\section{BiFC assay}

For construction of plasmids used for BiFC assays, the entire coding regions of SCL28 and AtSMOS1 were amplified by PCR, and cloned into donor vectors pDONR201 and pDONR207 using BP Clonase II (Thermo Fisher), respectively. The cloned fragments were then transferred 
into the destination vectors pGWnY and pGWcY (Hino et al. 2011) using LR clonase II (Thermo Fisher) to generate C-terminal fusions of YFP fragments. Transient gene expression using Arabidopsis leaf mesophyll protoplasts was performed as described previously (Yoshida et al. 2014). The transfected protoplasts were incubated overnight in the dark at $22^{\circ} \mathrm{C}$. YFP fluorescent was observed with fluorescent optics on a BX54 microscope (Olympus).

\section{LUC reporter assay}

Preparation of protoplasts from Arabidopsis T87 suspension cultured cells and polyethylene glycol-mediated gene transfer were performed as described previously (Maeo et al. 2009). An empty vector containing 35S promoter (pJIT-60) and 35S:hRLUC plasmid expressing the humanized Renilla LUC (hRLUC) were used as a negative control and an internal control, respectively. Protoplasts $\left(1.5 \times 10^{5}\right.$ for each transfection $)$ were co-transfected with $15 \mu \mathrm{g}$ each of the $L U C$ reporter and expression plasmids, then incubated at $22^{\circ} \mathrm{C}$ for $20 \mathrm{~h}$ before measuring LUC activities, which was performed using the Dual-Luciferase Reporter Assay system (Promega) and Luminoskan Ascent luminometer (Thermo Fisher). LUC activity was normalized according to hRLUC activity in each assay, and the relative ratio was determined.

\section{Plasmid construction}

To construct GUS fusion reporters, the upstream region of $S C L 28(2.2 \mathrm{~kb})$ was amplified by PCR and cloned into the pENTR/D-TOPO vector (Thermo Fisher), then transferred to pBGGUS (Haga et al., 2011) through Gateway LR reaction to create proSCL28::GUS. The PCR-based site-directed mutagenesis was performed to change all four MSA core motives, AACGG, in the SCL28 promoter to ATTGG, resulting in generation of proSCL28 $\triangle \mathrm{MSA}:$ GUS.

To prepare the construct expressing SCL28 fused to GFP at its C-terminus (SCL28-GFP) under the control of the native promoter, the entire $S C L 28$ genomic region $(5.3 \mathrm{~kb})$ containing 2.2-kb promoter was amplified by PCR using genomic DNA from Arabidopsis (Col), and cloned into pENTR/D-TOPO. The resulting plasmid was then used for In-Fusion reaction (Takara Clontech) to insert PCR-amplified GFP fragments at the C-terminus of the SCL28 coding sequence, to create entry plasmid containing the proSCL28::SCL28-GFP fusion construct, which was then transferred to the binary vector pGWB501 (Nakagawa et al., 2007).

To prepare the AtSMOS1-GFP fusion construct driven by its own promoter, the entire AtSMOS1 genomic region $(4.4 \mathrm{~kb})$ containing $1.2-\mathrm{kb}$ promoter was amplified by PCR using genomic DNA from Arabidopsis (Col) and cloned into pDONR201 through Gateway BP reaction (Thermo Fisher). The resulting plasmid was then used for In-Fusion reaction (Takara Clontech) 
for inserting PCR-amplified GFP fragment at the C-terminus of AtSMOS1, to create entry plasmid containing proAtSMOS1::AtSMOS1-GFP fusion construct, which was then transferred to the binary vector pPZP211-GW (Kozgunova et al., 2016).

For construction of a LUC reporter plasmid, promoter region of $S M R 2(2.0 \mathrm{~kb})$ was amplified by PCR and cloned into HindIII-BamHI interval of pUC-LUC (Ito et al., 2001) to obtain proSMR2::LUC. For construction of expression plasmids of AtSMOS1 and SCL28 (pro35S::AtSMOS1 and pro35S::SCL28), the entire coding regions were amplified by PCR using cDNA prepared from Arabidopsis T87 cells as a template. The resulting PCR fragments were cloned into pENTR/D-TOPO and then transferred to pJIT60 (Ito et al., 2001) at the site downstream of $35 \mathrm{~S}$ promoter through the Gateway LR reaction.

To create proRPS5A::SCL28-GFP, the entire coding sequence of SCL28-GFP fusion was amplified by PCR using proSCL28::SCL28-GFP plasmid as a template and cloned into pENTR/D-TOPO. The resulting plasmid was then used for the Gateway LR reaction to transfer the SCL28-GFP fragment downstream of RPS5A promoter in pPZP221 (Haga et al., 2007). Primers used for plasmid construction are listed in Supplementary Table 1.

\section{Histological analysis}

Excised plant organs were fixed in FAA solution (100\% ethanol: formaldehyde: glacial acetic acid: water $=20: 19: 1: 1)$ under vacuum, stained with $1 \%$ Safranin O, dehydrated with 30\%, 50\%, $70 \%$, and 100\% ethanol series, and embedded in Technovit 7100 (Heraeus Kulzer). $2 \mu \mathrm{m}$ thickness sections were cut on RM2125RT microtome (Leica) equipped with a tungsten carbide disposable blade TC-65 (Leica). Sections were briefly stained in a $0.01 \%-0.5 \%$ (w/v) toluidine blue-O in $0.1 \%$ (w/v) $\mathrm{Na}_{2} \mathrm{CO}_{3}$ solution, then washed with $0.1 \% \mathrm{Na}_{2} \mathrm{CO}_{3}$ solution. Sections were observed under BX63 microscope (Olympus) and images were acquired with cellSens Standard Software (Olympus).

\section{Chromatin immunoprecipitation assay}

ChIP-Seq assays were performed on whole seedlings using anti-GFP antibody (Clontech 632592). Seedlings at 14 DAS (5 g) from proAtSMOS1::AtSMOS1-GFP and proSCL28::SCL28-GFP were crosslinked in $1 \%(\mathrm{v} / \mathrm{v})$ formaldehyde at room temperature for $15 \mathrm{~min}$. Crosslinking was then quenched with $0.125 \mathrm{M}$ glycine for $5 \mathrm{~min}$. The crosslinked seedlings were ground, and nuclei were isolated and lysed in nuclei lysis buffer (1\% SDS, $50 \mathrm{mM}$ Tris-HCl, $10 \mathrm{mM}$ EDTA, pH 8.0). Crosslinked chromatin was sonicated using a water bath Bioruptor UCD-200 (Diagenode) (15sec on/15sec off pulses; 15 times). The complexes were immunoprecipitated with antibodies, 
overnight at $4{ }^{\circ} \mathrm{C}$ with gentle shaking, and incubated for $1 \mathrm{~h}$ at $4^{\circ} \mathrm{C}$ with $40 \mu \mathrm{L}$ of Protein AG UltraLink Resin (Thermo Fisher). The beads were washed $2 \times 5$ min in ChIP Wash Buffer 1 (0.1\% SDS, $1 \%$ Triton X-100, 20 mM Tris-HCl, 2 mM EDTA, $150 \mathrm{mM} \mathrm{NaCl}, \mathrm{pH} 8.0), 2 \times 5 \mathrm{~min}$ in ChIP Wash Buffer 2 (0.1\% SDS, 1\% Triton X-100, 20 mM Tris-HCl, 2 mM EDTA, 500 mM $\mathrm{NaCl}, \mathrm{pH} 8.0), 2 \times 5 \mathrm{~min}$ in ChIP Wash Buffer $3(0.25 \mathrm{M} \mathrm{LiCl}, 1 \% \mathrm{NP}-40,1 \%$ sodium deoxycholate, $10 \mathrm{mM}$ Tris-HCl, $1 \mathrm{mM}$ EDTA, $\mathrm{pH}$ 8.0) and twice in TE (10 mM Tris-HCl, $1 \mathrm{mM}$ EDTA, pH 8.0). ChIPed DNA was eluted by two 15 -min incubations at $65^{\circ} \mathrm{C}$ with $250 \mu \mathrm{L}$ elution buffer (1\% SDS, $0.1 \mathrm{M} \mathrm{NaHCO}_{3}$ ). Chromatin was reverse-crosslinked by adding $20 \mu \mathrm{L}$ of $5 \mathrm{M}$ $\mathrm{NaCl}$ and incubated overnight at $65^{\circ} \mathrm{C}$. Reverse-crosslinked DNA was subjected to RNase and proteinase $\mathrm{K}$ digestion and extracted with phenol-chloroform. DNA was ethanol precipitated in the presence of $20 \mu \mathrm{g}$ of glycogen and resuspended in $50 \mu \mathrm{L}$ of nuclease-free water (Ambion) in a DNA low-bind tube. We used $10 \mathrm{ng}$ of IP or input DNA for ChIP-Seq library construction using NEBNext ${ }^{\circledR}$ Ultra DNA Library Prep Kit for Illumina ${ }^{\circledR}$ (New England Biolabs) according to manufacturer's recommendations. For all libraries, twelve cycles of PCR were used. Library quality was assessed with Agilent 2100 Bioanalyzer (Agilent).

\section{Computational analysis of ChIP-Seq}

Single-end sequencing of ChIP samples was performed using Illumina NextSeq 500 with a read length of 76 bp. Reads were quality controlled using FASTQC (http://www.bioinformatics.babraham.ac.uk/projects/fastqc/). Trimmomatic was used for quality trimming. Parameters for read quality filtering were set as follows: minimum length of $36 \mathrm{bp}$; mean Phred quality score greater than 30; and leading and trailing bases removal with base quality below 5. Reads were mapped onto the TAIR10 assembly using Bowtie (Langmead and Salzberg, 2012) with mismatch permission of $1 \mathrm{bp}$. To identify significantly enriched regions, we used MACS2 (Zhang et al., 2008). Parameters for peak detection were set as follows: number of duplicate reads at a location: 1; mfold of 5: 50; q-value cutoff: 0.05; extsize 200; and sharp peak. Visualization and analysis of genome-wide enrichment profiles were performed with IGB. Peak annotations such as proximity to genes and overlap of genomic features, including transposons and genes were performed using BEDTOOLS INTERSECT. NGSplot was used to profile enrichment at TSSs and along the gene (Shen et al., 2014). Spatial binding of the AtSMOS1 and SCL28 peaks were performed by position-wise comparison using a binning approach and plotted in hexplot. De novo motif analysis of both SCL28 and AtSMOS1 binding regions were screened using HOMER (Kim et al., 2020). ChIP-Seq data of SCL28 and AtSMOS1 can be accessed at Gene Expression Omnibus database under accession number GSE183209. 


\section{Kinematic analysis of leaf growth}

After plants were fixed in a 9:1 of ethanol and acetic acid solution, and cleared with Hoyer's solution (a mixture of $100 \mathrm{~g}$ chloral hydrate, $10 \mathrm{~g}$ glycerol, $15 \mathrm{~g}$ gum arabic, and $25 \mathrm{ml}$ water), we performed microscopic observations using 1st or 2nd leaves as described previously (Haga et al. 2007). After whole leaf images were captured, palisade cells at positions one-fourth and threefourth from the tip of each leaf were observed with differential interference contrast (DIC) microscope (BX51, Olympus). The captured images were analyzed using ImageJ (ver.2.1.0; rsb.info.nih.gov/ij) and the average size of palisade cell, the number of cells in the uppermost layer of palisade tissue per leaf, and cell division rate were calculated according to methods described previously (De Veylder et al. 2001).

\section{Ploidy analysis}

For ploidy analysis, nuclei were isolated from whole leaves from plants at 8-20 DAS, then stained using CyStain UV precise P kit (Sysmex). After filtration, samples were analyzed with CyFlow Ploidy Analyser (Sysmex) according to the manufacturer's instructions. The population of nuclei in each peak was estimated as described previously (Kobayashi et al. 2015).

\section{Analysis of meristem cell number and cell size in roots}

To visualize cell outlines, roots from plants at 5DAS were stained with $0.05 \mathrm{mg}$ ml-1 propidium iodide and observed by confocal laser scanning microscopy (CLSM), using an inverted fluorescence microscope (Eclipse Ti2, Nikon) equipped with a confocal scanning unit (A1, Nikon). The resulting images were processed using ImageJ software to measure cell length in the root meristem. Root meristem size was measured by counting the number of cortical cells between the quiescent center and the first elongated cell.

\section{Cell cycle analysis by time-lapse imaging}

Seedlings at 6 DAS carrying PCNA-GFP were transferred onto an MS medium in a glass-bottom dish, and observed by CLSM as described above. Time-lapse images were acquired every $30 \mathrm{~min}$ for $15 \mathrm{~h}$. To avoid long-time imaging that possibly damages the samples, the duration of the G1/S and $\mathrm{G} 2 / \mathrm{M}$ phases was measured separately.

\section{Other experimental procedures}

ChIP-qPCR experiments (Kobayashi et al., 2015) and GUS staining (Haga et al., 2007) were 
performed as described previously. Primers used for ChIP-qPCR are listed in Supplementary Table 1.

\section{Data availability}

Arabidopsis mutants and transgenic lines, as well as plasmids generated in this study are available from the corresponding author upon reasonable request.

\section{Reference}

Andriankaja, M., Dhondt, S., De Bodt, S., Vanhaeren, H., Coppens, F., De Milde, L., Mühlenbock, P., Skirycz, A., Gonzalez, N., Beemster, G. T. \& Inzé, D. Exit from proliferation during leaf development in Arabidopsis thaliana: a not-so-gradual process. Dev. Cell 1764-78 (2012).

Aya, K., Hobo, T., Sato-Izawa, K., Ueguchi-Tanaka, M., Kitano, H. \& Matsuoka, M. A novel AP2-type transcription factor, SMALL ORGAN SIZE1, controls organ size downstream of an auxin signaling pathway. Plant Cell Physiol. 55, 897-912 (2014).

Barrada, A., Djendli, M., Desnos, T., Mercier, R., Robaglia, C., Montané, M. H. \& Menand, B. A TOR-YAK1 signaling axis controls cell cycle, meristem activity and plant growth in Arabidopsis. Development 146, dev171298.

Berckmans, B. \& De Veylder, L. Transcriptional control of the cell cycle. Curr. Opin. Plant Biol. 12, 599-605 (2009).

Bhosale, R., Boudolf, V., Cuevas, F., Lu, R., Eekhout, T., Hu, Z., Van Isterdael, G., Lambert, G. M., Xu, F., Nowack, M. K., Smith, R. S., Vercauteren, I., De Rycke, R., Storme, V., Beeckman, T., Larkin, J. C., Kremer, A., Höfte, H., Galbraith, D. W., Kumpf, R. P., Maere, S. \& De Veylder, L. A Spatiotemporal DNA Endoploidy Map of the Arabidopsis Root Reveals Roles for the Endocycle in Root Development and Stress Adaptation. Plant Cell 30, 2330-2351 (2018).

Borghi, L., Gutzat, R., Fütterer, J., Laizet, Y., Hennig, L. \& Gruissem, W. Arabidopsis RETINOBLASTOMA-RELATED is required for stem cell maintenance, cell differentiation, and lateral organ production. Plant Cell 22, 1792-1811 (2010).

Bouyer, D., Heese, M., Chen, P., Harashima, H., Roudier, F., Grüttner, C. \& Schnittger, A. Genome-wide identification of RETINOBLASTOMA RELATED 1 binding sites in Arabidopsis reveals novel DNA damage regulators. PLoS Genet. 14, e1007797 (2018). 
Choe, J., Kim, B., Yoon, E.K., Jang, S. Kim, G. Dhar, S., Lee, S.A. \& Lim, J. Characterization of the GRAS transcription factor SCARECROW-LIKE 28's role in Arabidopsis root growth. $J$ Plant Biol. 60, 462-471 (2017).

D'Ario, M., Tavares, R., Schiessl, K., Desvoyes, B., Gutierrez, C., Howard, M. \& Sablowski, R. Cell size controlled in plants using DNA content as an internal scale. Science 372, 1176-1181 (2021).

D'Ario, M. \& Sablowski, R. Cell Size Control in Plants. Annu. Rev. Genet. 53, 45-65 (2019).

Davie, E. \& Petersen, J. Environmental control of cell size at division. Curr. Opin. Cell Biol. 24, 838-844 (2012).

Desvoyes, B. \& Gutierrez, C. Roles of plant retinoblastoma protein: cell cycle and beyond. EMBO J.39, e105802 (2020).

De Veylder, L., Beeckman, T., Beemster, G. T., de Almeida Engler, J., Ormenese, S., Maes, S., Naudts, M., Van Der Schueren, E., Jacqmard, A., Engler, G. \& Inzé, D. Control of proliferation, endoreduplication and differentiation by the Arabidopsis E2Fa-DPa transcription factor. EMBO J. 21, 1360-1368 (2002).

De Veylder, L., Beeckman, T., Beemster, G. T. S., Krols, L., Terras, F., Landrieu, I., Van Der Schueren, E., Maes, S., Naudts, M. \& Inzé, D. Functional analysis of cyclin-dependent kinase inhibitors of Arabidopsis. Plant Cell 13, 1653-1667 (2001).

Dewitte, W., Riou-Khamlichi, C., Scofield, S., Healy, J. M., Jacqmard, A., Kilby, N. J. \& Murray, J. A. Altered cell cycle distribution, hyperplasia, and inhibited differentiation in Arabidopsis caused by the D-type cyclin CYCD3. Plant Cell 15, 79-92 (2003).

Fantes, P. A., Grant, W. D., Pritchard, R. H., Sudbery, P. E. \& Wheals, A. E. The regulation of cell size and the control of mitosis. J. Theor. Biol. 50, 213-244 (1975).

Ferjani, A., Horiguchi, G., Yano, S. \& Tsukaya, H. Analysis of leaf development in fugu mutants of Arabidopsis reveals three compensation modes that modulate cell expansion in determinate organs. Plant Physiol. 144, 988-999 (2007).

Fischer, M. \& Müller, G. A. Cell cycle transcription control: DREAM/MuvB and RB-E2F complexes. Crit. Rev. Biochem. Mol. Biol. 52, 638-662 (2017).

Goldy, C., Pedroza-Garcia, J. A., Breakfield, N., Cools, T., Vena, R., Benfey, P. N., De Veylder, L., Palatnik, J. \& Rodriguez, R. E. The Arabidopsis GRAS-type SCL28 transcription factor controls the mitotic cell cycle and division plane orientation. Proc. Natl. Acad.Sci.U S A.118, e2005256118 (2021).

Gutzat, R., Borghi, L., Fütterer, J., Bischof, S., Laizet, Y., Hennig, L., Feil, R., Lunn, J. \& Gruissem, W. RETINOBLASTOMA-RELATED PROTEIN controls the transition to 
autotrophic plant development. Development 138, 2977-2986 (2011).

Haga, N., Kato, K., Murase, M., Araki, S., Kubo, M., Demura, T., Suzuki, K., Müller, I., Voss, U., Jürgens, G. \& Ito, M. R1R2R3-Myb proteins positively regulate cytokinesis through activation of KNOLLE transcription in Arabidopsis thaliana. Development 134, 1101-1110 (2007).

Haga, N., Kobayashi, K., Suzuki, T., Maeo, K., Kubo, M., Ohtani, M., Mitsuda, N., Demura, T., Nakamura, K., Jürgens, G. \& Ito, M. Mutations in MYB3R1 and MYB3R4 cause pleiotropic developmental defects and preferential down-regulation of multiple G2/M-specific genes in Arabidopsis. Plant Physiol. 157, 706-17 (2011).

Hino, T., Tanaka, Y., Kawamukai, M., Nishimura, K., Mano, S. \& Nakagawa T. Two Sec13p homologs, AtSec13A and AtSec13B, redundantly contribute to the formation of COPII transport vesicles in Arabidopsis thaliana. Biosci. Biotechnol. Biochem. 75, 1848-1852 (2011).

Hirano, K., Yoshida, H., Aya, K., Kawamura, M., Hayashi, M., Hobo, T., Sato-Izawa, K., Kitano, H., Ueguchi-Tanaka, M. \& Matsuoka, M. SMALL ORGAN SIZE 1 and SMALL ORGAN SIZE 2/DWARF AND LOW-TILLERING Form a Complex to Integrate Auxin and Brassinosteroid Signaling in Rice. Mol. Plant 10, 590-604 (2017).

Hisanaga, T., Kawade, K. \& Tsukaya, H. Compensation: a key to clarifying the organ-level regulation of lateral organ size in plants. J. Exp. Bot. 66, 1055-1063 (2015).

Ito, M., Araki, S., Matsunaga, S., Itoh, T., Nishihama, R., Machida, Y., Doonan, J. H. \& Watanabe, A. G2/M-phase-specific transcription during the plant cell cycle is mediated by c-Myb-like transcription factors. Plant Cell 13, 1891-905 (2001).

Ito, M., Iwase, M., Kodama, H., Lavisse, P., Komamine, A., Nishihama, R., Machida, Y. \& Watanabe, A. A novel cis-acting element in promoters of plant B-type cyclin genes activates M phase-specific transcription. Plant Cell 10, 331-341 (1998).

Ito, M., Kodama, H. \& Komamine, A. Gene expression and its regulation during the cell cycle of higher plants in synchronous cell culture systems. In Vitro Cell. Dev. Biol. 35, 388-395 (1999). Jones, A.R., Band, L.R. \& Murray, J. A. H. Double or Nothing? Cell Division and Cell Size Control. Trends Plant Sci. 24, 1083-1093 (2019).

Jones, A. R., Forero-Vargas, M., Withers, S. P., Smith, R. S., Traas, J., Dewitte, W. \& Murray, J. A. H. Cell-size dependent progression of the cell cycle creates homeostasis and flexibility of plant cell size. Nat. Commun. 8, 15060 (2017).

Kim, S., Piquerez, S. J. M., Ramirez-Prado, J. S., Mastorakis, E., Veluchamy, A., Latrasse, D., Manza-Mianza, D., Brik-Chaouche, R., Huang, Y., Rodriguez-Granados, N. Y., Concia, L., Blein, T., Citerne, S., Bendahmane, A., Bergounioux, C., Crespi, M., Mahfouz, M. M., 
Raynaud, C., Hirt, H., Ntoukakis, V. \& Benhamed, M. GCN5 modulates salicylic acid homeostasis by regulating $\mathrm{H} 3 \mathrm{~K} 14 \mathrm{ac}$ levels at the $5^{\prime}$ and $3^{\prime}$ ends of its target genes. Nucleic Acids Res. 48, 5953-5966 (2020).

Kobayashi, K., Suzuki, T., Iwata, E., Nakamichi, N., Suzuki, T., Chen, P., Ohtani, M., Ishida, T., Hosoya, H., Müller, S., Leviczky, T., Pettkó-Szandtner, A., Darula, Z., Iwamoto, A., Nomoto, M., Tada, Y., Higashiyama, T., Demura, T., Doonan, J. H., Hauser, M. T., Sugimoto, K., Umeda, M., Magyar, Z., Bögre, L. \& Ito M. Transcriptional repression by MYB3R proteins regulates plant organ growth. EMBO J. 34, 1992-2007 (2015).

Komaki, S. \& Sugimoto, K. Control of the plant cell cycle by developmental and environmental cues. Plant Cell Physiol. 53, 953-964 (2012).

Kozgunova, E., Suzuki, T., Ito, M., Higashiyama, T. \& Kurihara, D. Haspin has Multiple Functions in the Plant Cell Division Regulatory Network. Plant Cell Physiol. 57, 848-861 (2016).

Kumar, N., Harashima, H., Kalve, S., Bramsiepe, J., Wang, K., Sizani, B. L., Bertrand, L. L., Johnson, M. C., Faulk, C., Dale, R., Simmons, L. A., Churchman, M. L., Sugimoto, K., Kato, N., Dasanayake, M., Beemster, G., Schnittger, A. \& Larkin, J. C. Functional conservation in the SIAMESE-RELATED family of cyclin-dependent kinase inhibitors in land plants. Plant Cell 27, 3065-3080 (2015).

Lang, L., Pettkó-Szandtner, A., Tunçay Elbaşı, H., Takatsuka, H., Nomoto, Y., Zaki, A., Dorokhov, S., De Jaeger, G., Eeckhout, D., Ito, M., Magyar, Z., Bögre, L., Heese, M., \& Schnittger, A. The DREAM complex represses growth in response to DNA damage in Arabidopsis. Life Sci. Alliance 4, e202101141 (2021).

Langmead, B. \& Salzberg, S. L. Fast gapped-read alignment with Bowtie 2. Nat. Methods 9, 357359 (2012).

Lee, T. I., Rinaldi, N. J., Robert, F., Odom, D. T., Bar-Joseph, Z., Gerber, G. K., Hannett, N. M., Harbison, C. T., Thompson, C. M., Simon, I., Zeitlinger, J., Jennings, E. G., Murray, H. L., Gordon, D. B., Ren, B., Wyrick, J. J., Tagne, J. B., Volkert, T. L., Fraenkel, E., Gifford, D. K. \& Young, R. A. Transcriptional regulatory networks in Saccharomyces cerevisiae. Science 298, 799-804 (2002).

Maeo, K., Tokuda, T., Ayame, A., Mitsui, N., Kawai, T., Tsukagoshi, H., Ishiguro, S. \& Nakamura, K. An AP2-type transcription factor, WRINKLED1, of Arabidopsis thaliana binds to the AW-box sequence conserved among proximal upstream regions of genes involved in fatty acid synthesis. Plant J. 60, 476-487 (2009).

Magyar, Z., De Veylder, L., Atanassova, A., Bakó, L., Inzé, D. \& Bögre, L. The role of the 
Arabidopsis E2FB transcription factor in regulating auxin-dependent cell division. Plant Cell 17, 2527-2541 (2005).

Meyer, H. M., Teles, J., Formosa-Jordan, P., Refahi, Y., San-Bento, R., Ingram, G., Jönsson, H., Locke, J. C. \& Roeder, A. H. Fluctuations of the transcription factor ATML1 generate the pattern of giant cells in the Arabidopsis sepal. Elife 6, e19131 (2017).

Millour, J., de Olano, N., Horimoto, Y., Monteiro, L.J., Langer, J.K., Aligue, R., Hajji, N. \& Lam, E.W. ATM and p53 regulate FOXM1 expression via E2F in breast cancer epirubicin treatment and resistance. Mol. Cancer Ther. 10, 1046-1058 (2011).

Nakagawa, T., Suzuki, T., Murata, S., Nakamura, S., Hino, T., Maeo, K., Tabata, R., Kawai, T., Tanaka, K., Niwa, Y., Watanabe, Y., Nakamura, K., Kimura, T. \& Ishiguro, S. Improved Gateway binary vectors: high-performance vectors for creation of fusion constructs in transgenic analysis of plants. Biosci Biotechnol Biochem. 71, 2095-2100 (2007).

Ning, Y. Q., Liu, N., Lan, K. K., Su, Y. N., Li, L., Chen, S. \& He, X. J. DREAM complex suppresses DNA methylation maintenance genes and precludes DNA hypermethylation. Nat. Plants 6, 942-956 (2020).

Őszi, E., Papdi, C., Mohammed, B., Petkó-Szandtner, A., Leviczky, T., Molnár, E., GalvanAmpudia, C., Khan, S., Juez, E. L., Horváth, B., Bögre, L. \& Magyar Z. E2FB Interacts with RETINOBLASTOMA RELATED and Regulates Cell Proliferation during Leaf Development. Plant Physiol. 182, 518-533 (2020).

Okumura, T., Nomoto, Y., Kobayashi, K., Suzuki, T., Takatsuka, H. \& Ito M. MYB3R-mediated active repression of cell cycle and growth under salt stress in Arabidopsis thaliana. J. Plant Res. 134, 261-277 (2021).

O'Malley, R. C., Huang, S. C., Song, L., Lewsey, M. G., Bartlett, A., Nery, J. R., Galli, M., Gallavotti, A. \& Ecker J. R. Cistrome and Epicistrome Features Shape the Regulatory DNA Landscape. Cell 165, 1280-1292 (2016).

Pavelescu, I., Vilarrasa-Blasi, J., Planas-Riverola, A., González-García, M. P., Caño-Delgado, A. I. \& Ibañes, M. A Sizer model for cell differentiation in Arabidopsis thaliana root growth. Mol Syst Biol. 14, e7687 (2018).

Polyn, S., Willems, A. \& De Veylder, L. Cell cycle entry, maintenance, and exit during plant development. Curr. Opin. Plant Biol. 23, 1-7 (2015).

Potter, C. J. \& Xu, T. Mechanisms of size control. Curr. Opin. Genet. Dev. 11, 279-86 (2001). Qi, F. \& Zhang, F. Cell Cycle Regulation in the Plant Response to Stress. Front. Plant Sci. 10, 1765 (2020).

Roeder, A. H., Chickarmane, V., Cunha, A., Obara, B., Manjunath, B. S. \& Meyerowitz, E. M. Variability in the control of cell division underlies sepal epidermal patterning in Arabidopsis 
thaliana. PLoS Biol. 8, e1000367 (2010).

Sadasivam, S. \& DeCaprio, J. A. The DREAM complex: master coordinator of cell cycledependent gene expression. Nat. Rev. Cancer. 13, 585-95 (2013).

Schmoller, K. M., Turner, J. J., Kõivomägi, M. \& Skotheim, J. M. Dilution of the cell cycle inhibitor Whi5 controls budding-yeast cell size. Nature 526, 268-272 (2015).

Shen, L., Shao, N., Liu, X. \& Nestler, E. ngs.plot: Quick mining and visualization of nextgeneration sequencing data by integrating genomic databases. BMC Genomics, 15, 284 (2014)

Simon, I., Barnett, J., Hannett, N., Harbison, C. T., Rinaldi, N. J., Volkert, T. L., Wyrick, J. J., Zeitlinger, J., Gifford, D. K., Jaakkola, T. S. \& Young, R. A. Serial regulation of transcriptional regulators in the yeast cell cycle. Cell 106, 697-708 (2001).

Soyano, T., Nishihama, R., Morikiyo, K., Ishikawa, M. \& Machida Y. NQK1/NtMEK1 is a MAPKK that acts in the NPK1 MAPKKK-mediated MAPK cascade and is required for plant cytokinesis. Genes Dev. 17, 1055-67 (2003).

Ubeda-Tomás, S., Federici, F., Casimiro, I., Beemster, G. T. S., Bhalerao, R., Swarup, R., Doerner, P., Haseloff, J. \& Bennett, M. J. Gibberellin signaling in the endodermis controls Arabidopsis root meristem size. Curr. Biol. 19, 1194-1199 (2009).

Vandepoele, K., Vlieghe, K., Florquin, K., Hennig, L., Beemster, G. T., Gruissem, W., Van de Peer, Y., Inzé, D. \& De Veylder L. Genome-wide identification of potential plant E2F target genes. Plant Physiol. 139, 316-328 (2005).

Verkest, A., Manes, C. L., Vercruysse, S., Maes, S., Van Der Schueren, E., Beeckman, T., Genschik, P., Kuiper, M., Inzé, D. \& De Veylder, L. The cyclin-dependent kinase inhibitor KRP2 controls the onset of the endoreduplication cycle during Arabidopsis leaf development through inhibition of mitotic CDKA;1 kinase complexes. Plant Cell 17, 1723-1736 (2005).

Wang, K., Ndathe, R. W., Kumar, N., Zeringue, E. A., Kato, N. \& Larkin, J. C. The CDK Inhibitor SIAMESE Targets Both CDKA;1 and CDKB1 Complexes to Establish Endoreplication in Trichomes. Plant Physiol. 184, 165-175 (2020).

Willis, L., Refahi, Y., Wightman, R., Landrein, B., Teles, J., Huang, K. C., Meyerowitz, E. M. \& Jönsson, H. Cell size and growth regulation in the Arabidopsis thaliana apical stem cell niche. Proc. Natl. Acad. Sci. U S A. 113, E8238-E8246 (2016).

Wu, C. Y., Rolfe, P. A., Gifford, D. K. \& Fink, G. R. Control of transcription by cell size. PLoS Biol. 8, e1000523 (2010).

Yi, D., Alvim Kamei, C. L., Cools, T., Vanderauwera, S., Takahashi, N., Okushima, Y., Eekhout, T., Yoshiyama, K. O., Larkin, J., Van den Daele, H., Conklin, P., Britt, A., Umeda, M. \& De Veylder, L. The Arabidopsis SIAMESE-RELATED cyclin-dependent kinase inhibitors SMR5 
and SMR7 regulate the DNA damage checkpoint in response to reactive oxygen species. Plant Cell 26, 296-309 (2014).

Yokoyama, R., Hirakawa, T., Hayashi, S., Sakamoto, T. \& Matsunaga, S. Dynamics of plant DNA replication based on PCNA visualization. Sci.Rep. 6, 29657 (2016).

Yoshida, H., Hirano, K., Sato, T., Mitsuda, N., Nomoto, M., Maeo, K., Koketsu, E., Mitani, R., Kawamura, M., Ishiguro, S., Tada, Y., Ohme-Takagi, M., Matsuoka, M. \& Ueguchi-Tanaka, M. DELLA protein functions as a transcriptional activator through the DNA binding of the indeterminate domain family proteins. Proc. Natl. Acad. Sci. U S A 111, 7861-7866 (2014).

Zhang, Y., Liu, T., Meyer, C. A., Eeckhoute, J., Johnson, D. S., Bernstein, B. E., Nusbaum, C., Myers, R. M., Brown, M., Li, W. \& Liu, X. S. Model-based Analysis of ChIP-Seq (MACS). Genome Biol. 9, R137 (2008).

Zatulovskiy, E. \& Skotheim, J. M. On the Molecular Mechanisms Regulating Animal Cell Size Homeostasis. Trends Genet. 36, 360-372 (2020).

Zatulovskiy, E., Zhang, S., Berenson, D. F., Topacio, B. R. \& Skotheim, J. M. Cell growth dilutes the cell cycle inhibitor Rb to trigger cell division. Science 369, 466-471 (2020).

Zhu, W., Giangrande, P. H., Nevins, J. R. E2Fs link the control of G1/S and G2/M transcription. ЕМВО J. 23, 4615-26 (2004).

\section{Acknowledgements}

The authors thank Kyoko Kato, Akiko Nakanishi, Natsuko Ono, Akiko Yamamoto, Chikako Inoue, Nanako Ishibashi, Satoko Nasu, Yuiko Tachikawa, Asako Segawa, Ayami Furuta, Tomomi Shinagara and Ayumi Yamada for technical assistance. This study was funded by The Japan Society for the Promotion of Science KAKENHI (20H05408 and 17H03696 to M. Ito, 20H05911 and 20H05905 to K. Sugimoto), and bythe Institut Universitaire de France (to M. Benhamed). Y. Huang was supported by China Scholar Council fellowships (201806690005).

\section{Author contributions}

M. Ito. conceived the study; C.R., M.B., Z.M., K.S., T.Y., and M.I. designed the experiments; M. Imamura., K.M., T.I., C.B., M.G., D.L., Y.H., Toshiya Suzuki, K.Y., H.T., and Y.N. carried out the experiments; J.A., Takamasa Suzuki analyzed the data; M.I., L.B., Z.M., and M.B. wrote the paper

\section{Competing interests}


bioRxiv preprint doi: https://doi.org/10.1101/2021.10.16.464643; this version posted October 17, 2021. The copyright holder for this preprint (which was not certified by peer review) is the author/funder, who has granted bioRxiv a license to display the preprint in perpetuity. It is made available under aCC-BY-NC-ND 4.0 International license.

The authors declare no competing interests.

\section{Additional information}

Supplementary information The online version contains supplementary material. 
a
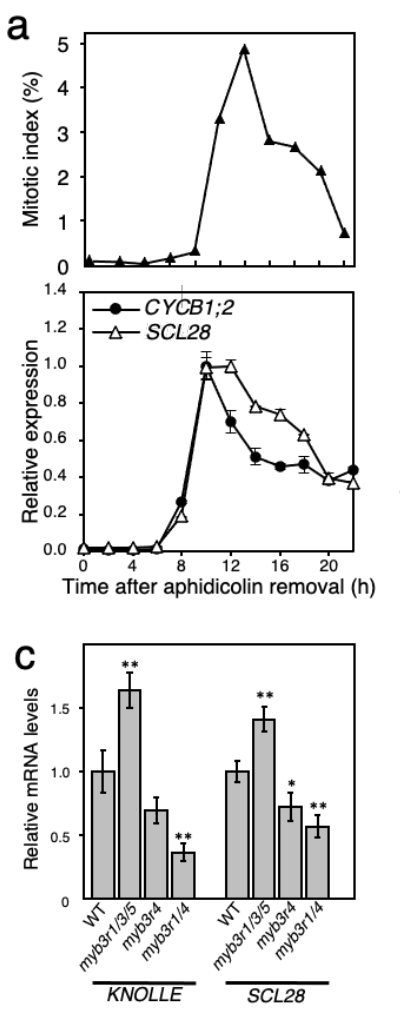

b

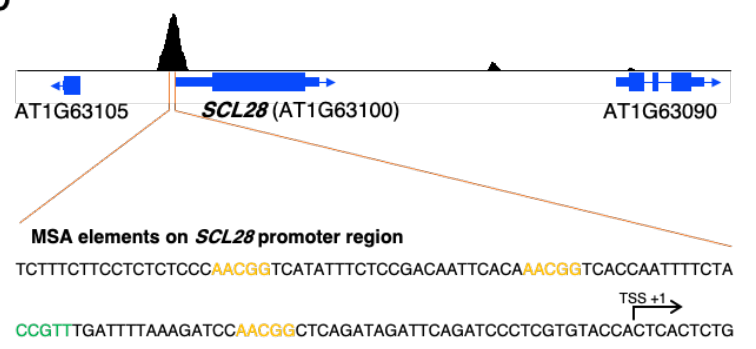

d
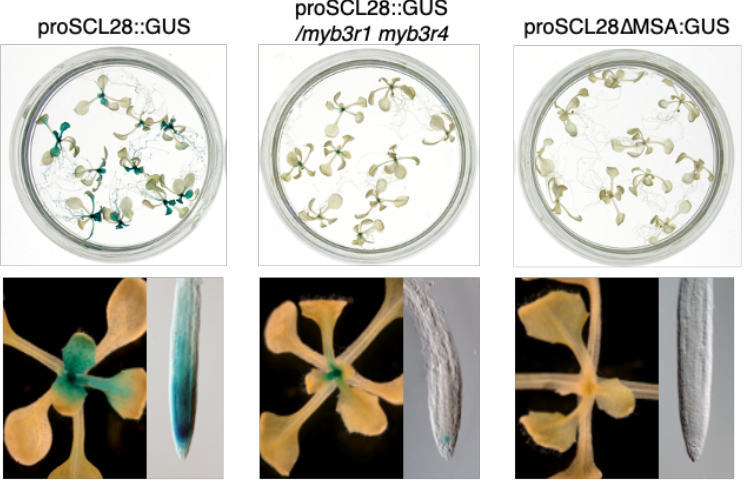

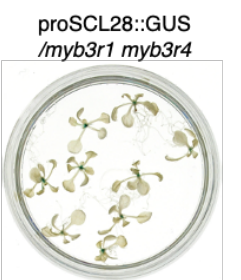

proSCL28 MMSA:GUS

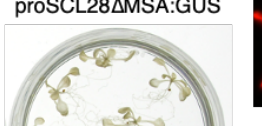

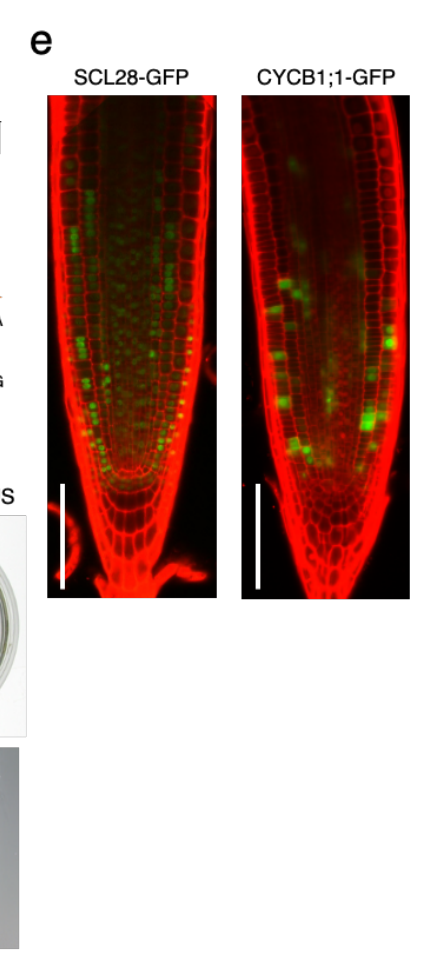

Fig. 1 SCL28 expression is cell cycle-regulated under the control of MYB3R transcription factors. a G2/M-specific accumulation of SCL28 transcript. Arabidopsis MM2d cells were synchronized by aphidicolin treatment, and used for qRT-PCR analysis to quantify SCL28 mRNA levels (lower). Synchronous progression of cell cycle was monitored by measuring mitotic index (upper). As a representative of G2/M-specific genes, $C Y C B 1$;2 was also analyzed in the same way for comparison. Expression data are shown as mean $\pm \mathrm{SD}(n=3)$.

b MYB3R3 binds to the upstream region of $S C L 28$. The ChIP-Seq profile of MYB3R3 indicates its direct binding to the proximal promoter region of SCL28. The nucleotide sequence around the ChIPSeq peak contains MSA elements repeated four times, of which core motives (AACGG) are shown by orange (forward orientation) and green (reverse orientation) letters.

c Levels of $S C L 28$ transcripts are significantly affected by mutations in myb3r genes. Transcript levels of SCL28 and KNOLLE were analyzed by qRT-PCR in plants 12 days after sowing (DAS) that carry mutations of $m y b 3 r$ genes in the indicated combinations (mean $\pm \mathrm{SD} ; n=3$ ). Statistical significance was determined using Student's t-test. $* P<0.05, * * P<0.01$.

d Promoter activity of SCL28 requires the presence of MYB3R activators and MSA elements. GUS reporter expression was analyzed in wild type (WT) and myb3r1/4 plants carrying proSCL28::GUS and WT plants carrying proSCL28 $\triangle \mathrm{MSA}:: G U S$, in which all MSA elements in the SCL28 promoter were mutated. GUS staining around shoot apex and root tip regions is shown at higher magnification in the lower panels. 
e Patchy pattern expression of SCL28-GFP protein. Root meristems of proSCL28::SCL28-GFP and proCYCB1;1::CYCB1;1-GFP plants were analyzed by confocal laser scanning microscopy (CLSM) after counterstaining of the cell wall with propidium iodide (PI). Green and red signals indicate fluorescence of GFP and PI, respectively. Scale bar indicates $100 \mu \mathrm{m}$. 
a

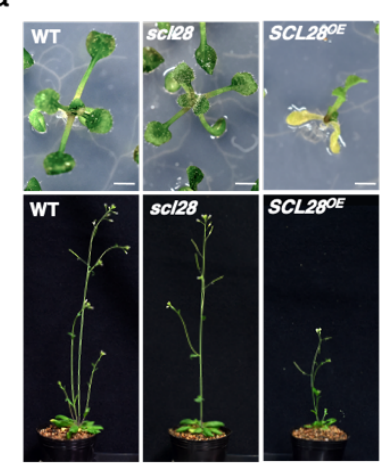

d

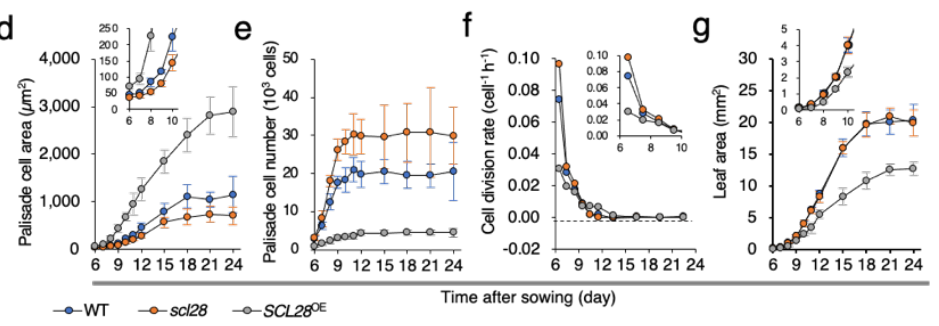

b WT

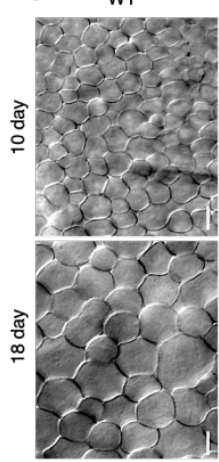

scl28

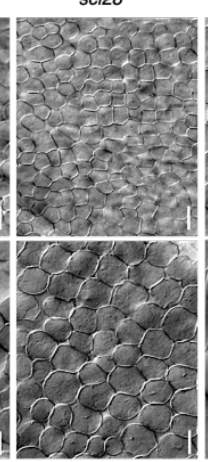

g

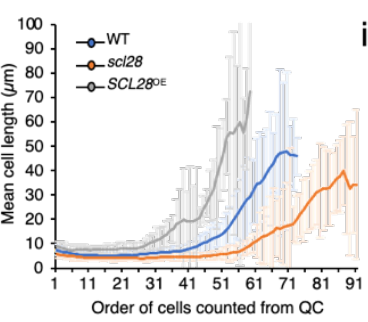

SCL28OE

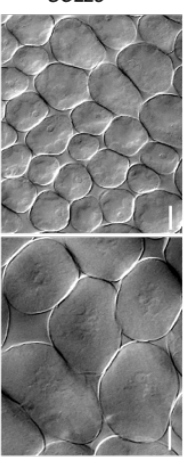

Order of cells counted from QC
C
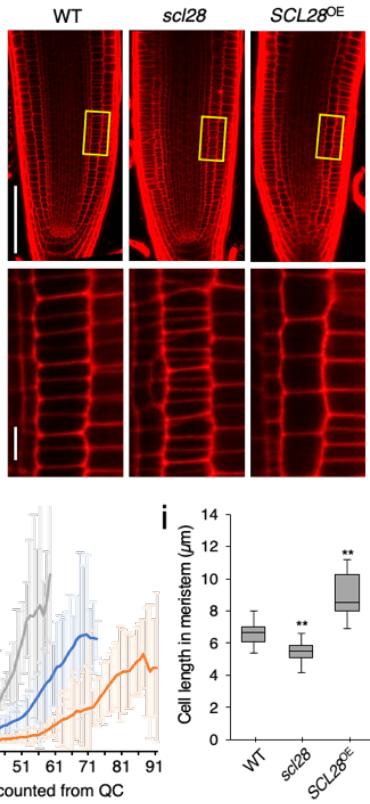

Fig. 2 SCL28 strongly affects cell size.

a Macroscopic phenotypes caused by loss- and gain-of-function of SCL28. WT, scl28, and SCL28 plants were photographed at 11 (upper) and 30 (lower) DAS. Scale bar in upper panels indicates $10 \mathrm{~mm}$. b Cell size analysis of leaf palisade tissue from WT, scl28, and $S C L 28^{\mathrm{OE}}$ plants. First leaf pairs from plants at 10 or 18 DAS were cleared and analyzed by differential interference contrast (DIC) microscopy. Scale bar indicates $20 \mu \mathrm{m}$.

c Cell size analysis of root meristem from WT, scl28, and $S C L 28^{\mathrm{OE}}$ plants at 5 DAS. Primary roots were stained with PI, and analyzed by CLSM. Regions surrounded by yellow rectangles are shown at a higher magnification in the lower panels. Scale bars indicate $100 \mu \mathrm{m}$ (upper) and $10 \mu \mathrm{m}$ (lower).

d-g Kinematic analysis of leaf growth in WT, scl28, and SCL28 ${ }^{\circ}$ plants. Time-course changes of palisade cell area $(\mathbf{d})$, number of palisade cells per leaf $(\mathbf{e})$, cell division rate $(\mathbf{f})$, and leaf area $(\mathbf{g})$ were analyzed in developing first leaf pairs. Data are shown as mean $\pm \operatorname{SD}(n=10)$. Inset shows the same data with an expanded $y$-axis scale.

h Spatial distribution of cell size across root meristems in WT, scl28, and SCL28 ${ }^{\mathrm{OE}}$ plants at 5 DAS. Mean cell length was calculated at each position along the cortical cell file, which is defined by counts of cortical cells from the quiescent center (mean $\pm \mathrm{SD} ; n=21$ ).

i Quantitative analysis of cortical cell length within root meristems. Data are shown as boxplot, where 'boxes' represent the interquartile range (IQR) and whiskers extend to the largest and smallest observations within $1.5 \times \mathrm{IQR}$. Statistical significance compared with WT was determined using Student's t-test. $* * P<0.01(n=20)$. 

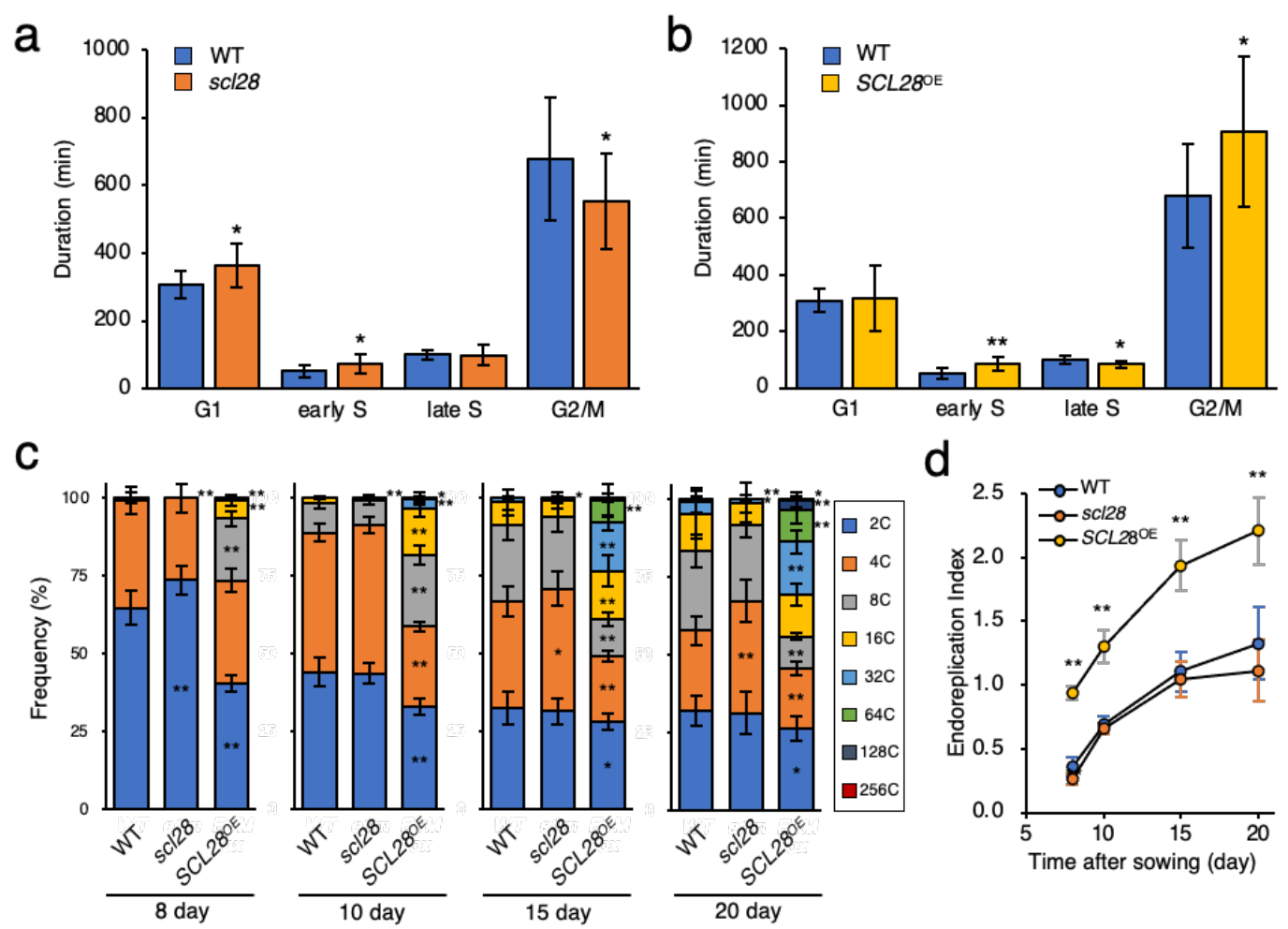

Fig. 3 SCL28 inhibits G2 progression and induces endoreplication.

a G2 duration is shortened in scl28. Cortical cells in root meristems of WT, scl28, and SCL28 ${ }^{\mathrm{OE}}$ plants carrying the PCNA-GFP marker were analyzed by live-cell imaging for measuring length of G1, early $\mathrm{S}$, late $\mathrm{S}$, and G2/M phases in the cell cycle. Data are shown as mean $\pm \mathrm{SD}(n \geq 10)$. Statistical significance compared with WT was determined using Student's t-test. ${ }^{*} P<0.05$.

b Prolonged G2 duration in SCL2 $8^{\mathrm{OE}}$ plants. Cell cycle analysis in the root meristem was performed as in (a). Data are shown as mean $\pm \mathrm{SD}(n \geq 10)$. Statistical significance compared with WT was determined using Student's t-test. $* P<0.05, * * P<0.01$.

c Ploidy analysis of $s c l 28$ and $S C L 28^{\mathrm{OE}}$ plants. First leaf pairs of WT, scl28, and SCL28 $8^{\mathrm{OE}}$ plants were subjected to flow cytometric analysis to determine ploidy distribution during leaf development. Data are shown as mean $\pm \mathrm{SD}(n=10)$. Statistical significance compared with WT was determined using Student's t-test. $* P<0.05, * * P<0.01$.

d Time-course change in cellular ploidy levels during leaf development. Data presented in (c) were used for calculating endoreplication index, which represents mean number of endoreplication cycles per leaf cells. Data are shown as mean $\pm \mathrm{SD}(n=10)$. Statistical significance compared with WT was determined using Student's t-test. $* * P<0.01$. 
a
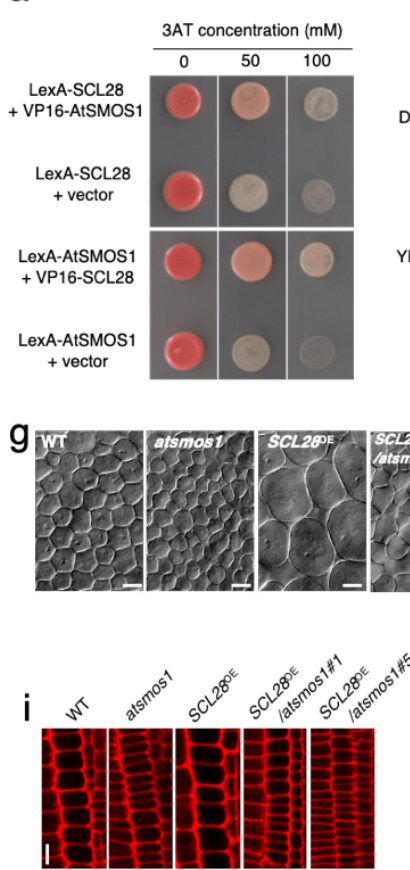

b
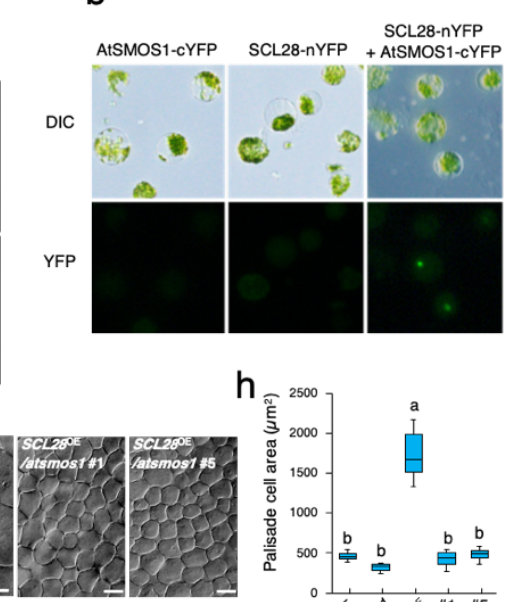

j

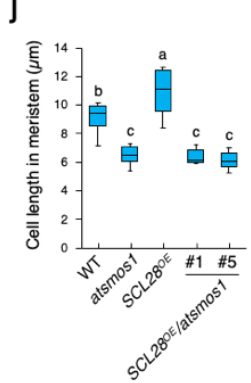

C

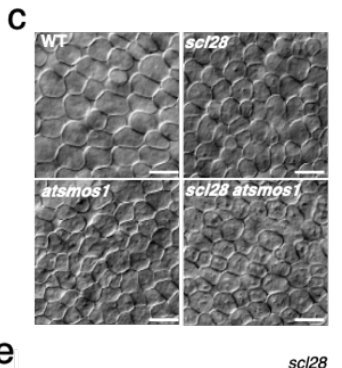

$\mathrm{e}_{\text {wr }}$
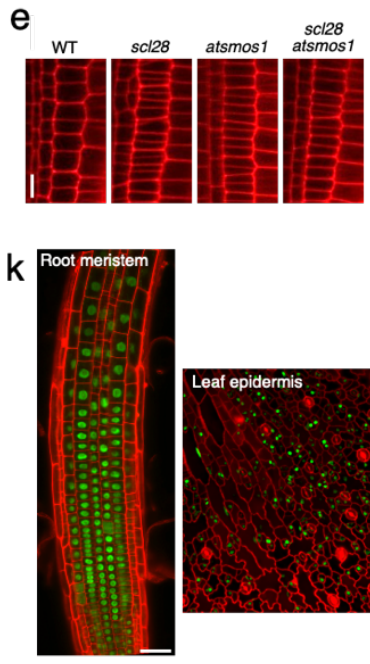

d
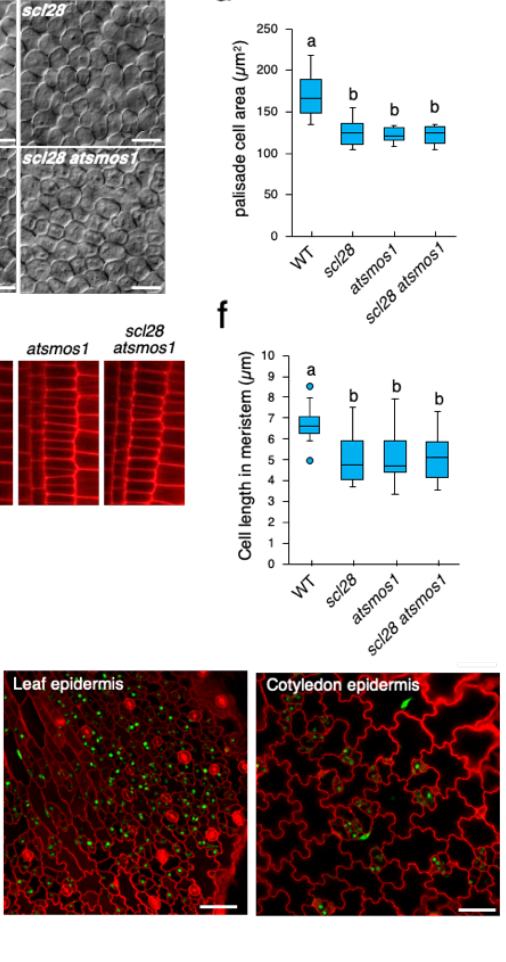

\section{Fig. 4 Formation of active heterodimer between SCL28 and AtSMOS1}

a SCL28-AtSMOS1 interaction in yeast two-hybrid assay. Yeast strains carrying two indicated constructs were grown on synthetic medium lacking His and containing indicated concentration of 3amino-1,2,4-triazole (3-AT).

b SCL28-AtSMOS1 interaction in bimolecular fluorescence complementation (BiFC) analysis. Protoplasts prepared from leaf mesophyll cells were transfected with the indicated plasmid constructs. Upper panels, DIC observation; lower panels, epifluorescence microscopic observation.

c Leaf palisade cells were observed by DIC microscopy using first leaf pairs from WT plants, and scl28, atsmos 1 , and double scl28 atsmos 1 mutants at 10 DAS. Scale bar indicates $20 \mu \mathrm{m}$.

d Quantification of palisade cell area in first leaf pairs from plants with indicated genotypes. Data are shown as boxplot $($ midline $=$ median, box $=\mathrm{IQR}$, whiskers $=1.5 \times \mathrm{IQR}$ ). Different letters above boxplots indicate significant differences based on one-way ANOVA and Tukey's test, $P<0.05(n=10)$. e Cortical cell files were observed by CLSM using PI-stained meristems of primary roots from WT plants, and scl28, atsmos 1 , and double scl28 atsmos 1 mutants at 5 DAS. Scale bars indicate $10 \mu \mathrm{m}$.

f Quantification of cortical cell length in root meristems from plants with indicated genotypes. Data are shown as boxplots as in (d). Different letters above boxplots indicate significant differences as in (d) (n $=20)$.

g DIC observation of palisade cells from first leaf pairs of WT, atsmos1, SCL28 ${ }^{\mathrm{OE}}$ plants, and those possessing atsmos 1 and SCL28 ${ }^{\circ \mathrm{E}}$ in combination. Plants at 15 DAS were used. Scale bar indicates 20 
$\mu \mathrm{m}$.

$\mathbf{h}$ Quantification of palisade cell area in first leaf pairs from the plants with indicated genotypes. Data are shown as boxplots as in (d). Different letters above the boxplots indicate significant differences as in $(\mathbf{d})(n=9)$.

i CLSM observation of PI-stained root meristems from WT, atsmos1, SCL28 ${ }^{\mathrm{OE}}$ plants, and those possessing atsmos 1 and SCL2 $8^{\mathrm{OE}}$ in combination. Plants at $8 \mathrm{DAS}$ were used for observation of cortical cell files. Scale bar indicates $10 \mu \mathrm{m}$.

j Quantification of cortical cell length in root meristems of plants with indicated genotypes. Data are shown as boxplots as in (d). Different letters above boxplots indicate significant differences as in (d) ( $n$ $\geq 5$ ).

k Accumulation patterns of AtSMOS1-GFP protein. Root meristem, and epidermis of leaf and cotyledon from plants at 6 DAS carrying proAtSMOS1::AtSMOS1-GFP were analyzed by CLSM after counterstaining of the cell wall with PI. Scale bars indicate $50 \mu \mathrm{m}$. 
a

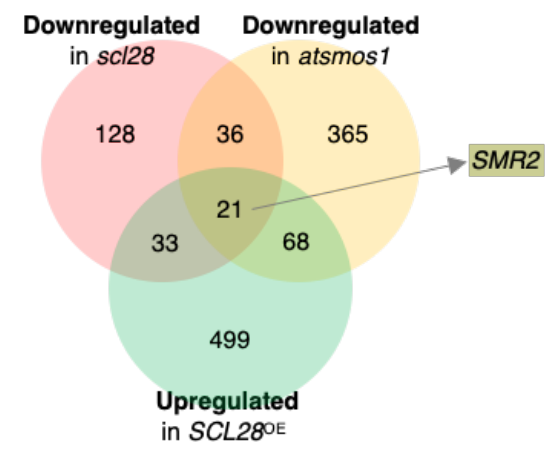

b

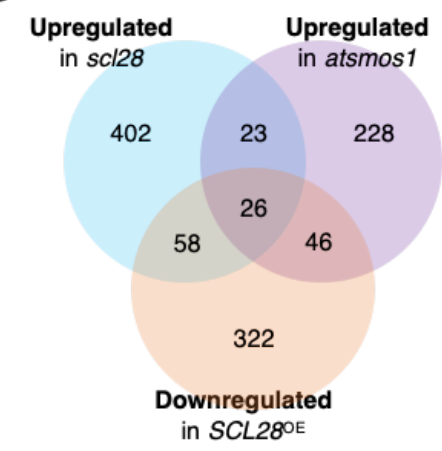

C

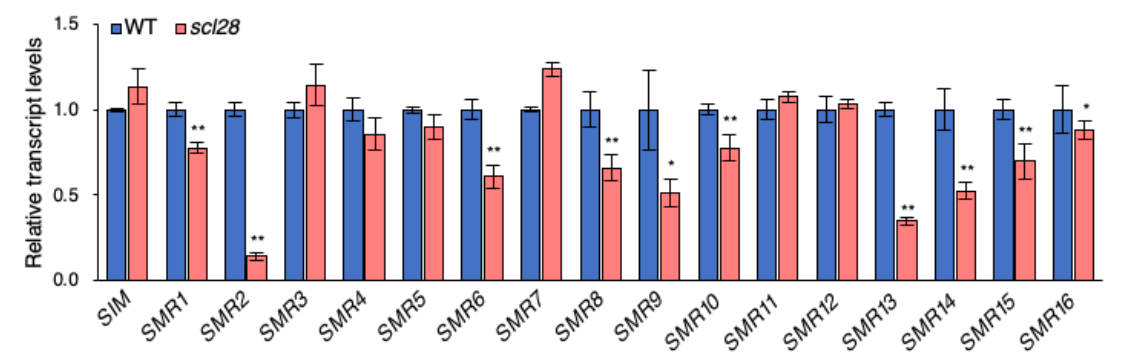

d

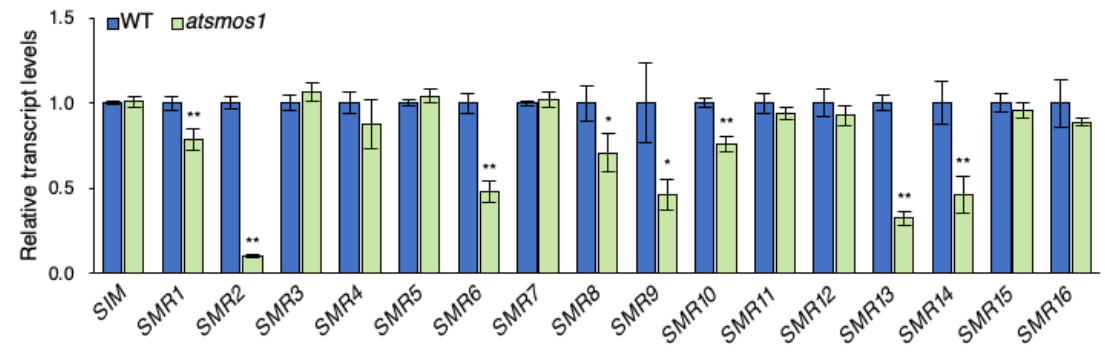

e

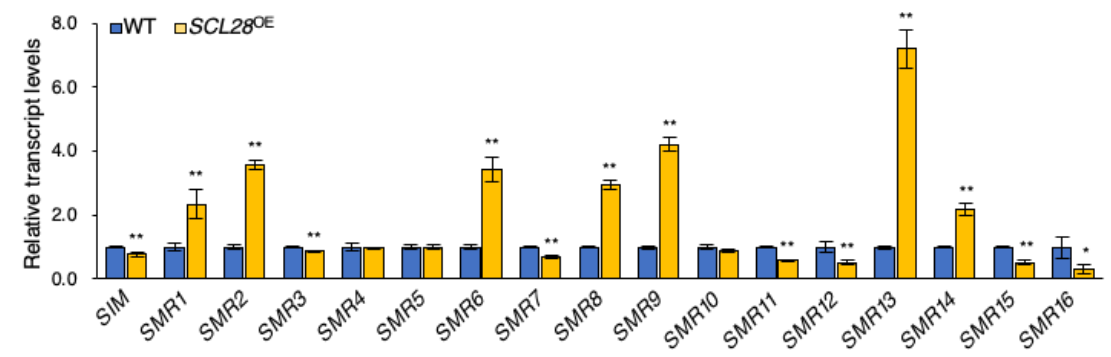

Fig. 5 SCL28 and AtSMOS1 activate transcription of SMR genes.

a Venn diagram representing overlap between genes downregulated in $s c l 28$ and atsmos 1 , and upregulated in $S C L 28^{\mathrm{OE}}$ plants. The $S M R 2$ gene was found in the overlap of these three categories.

b Venn diagram representing overlap between genes upregulated in scl28 and atsmos 1 , and downregulated in $S C L 28^{\mathrm{OE}}$ plants.

c-d Transcript levels of SMR genes in $\operatorname{scl} 28(\mathbf{c})$, atsmosl (d), and SCL28 ${ }^{\mathrm{OE}}$ (e) plants at 8 DAS. qRTPCR analysis of all 17 genes in the SMR family was performed to compare expression levels with WT plants. Data are shown as mean $\pm \mathrm{SD}(n=3)$. Statistical significance compared with WT was determined using Student's t-test. $* P<0.05, * * P<0.01$. 
a

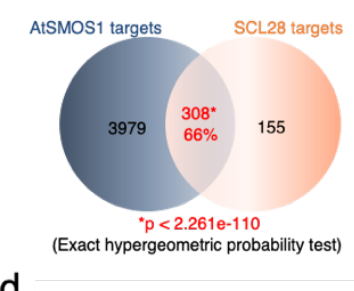

d

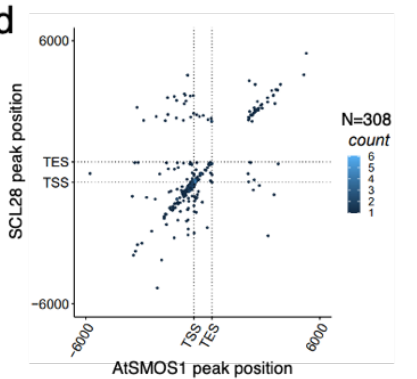

f

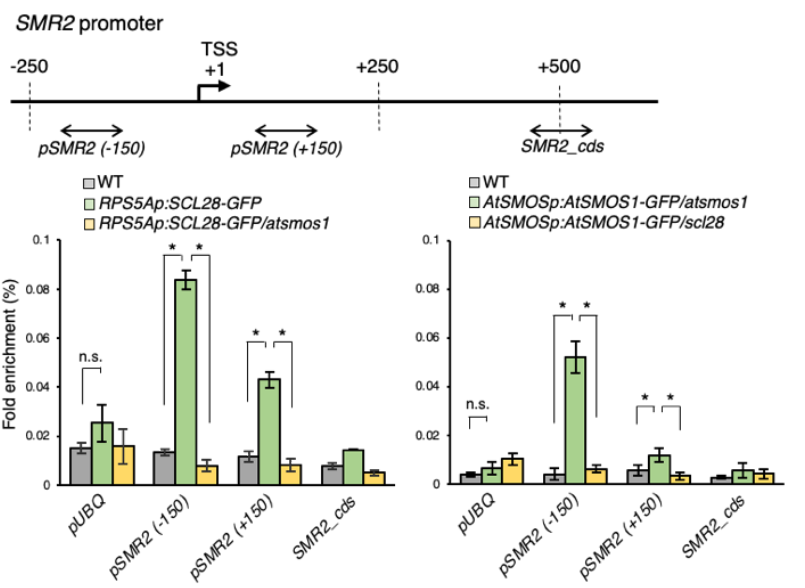

b

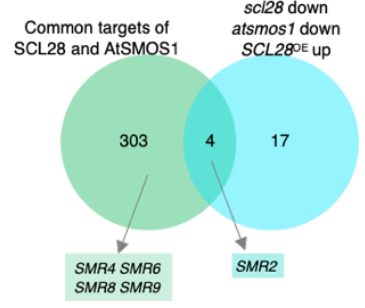

e

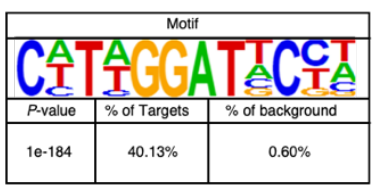

$50 \%$

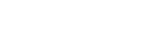


found in $40.13 \%$ of common targets.

f ChIP-qPCR analysis was performed on SMR2 loci using plants carrying proRPS5A::SCL28-GFP under WT and atsmos 1 backgrounds, and those carrying proAtSMOS1::AtSMOS1-GFP under scl28 and atsmos 1 backgrounds. Amplified regions $(-150,+150$, and cds) in ChIP-qPCR are shown by doubleheaded arrows. $U B Q$ locus was analyzed in the same way as a negative control without binding. Data are shown as mean $\pm \mathrm{SD}(n=3)$. Statistical significance was determined using Student's t-test. $* P<$ 0.05. n.s., not significant. 
a

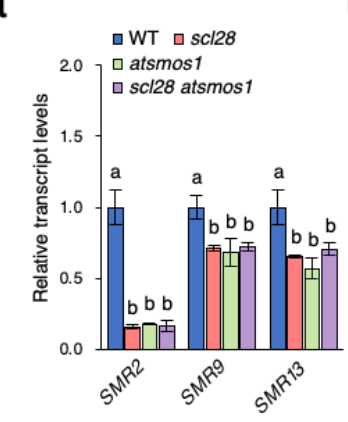

b

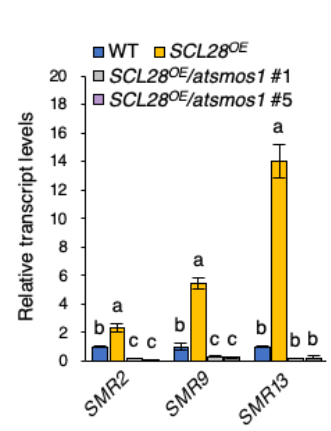

d

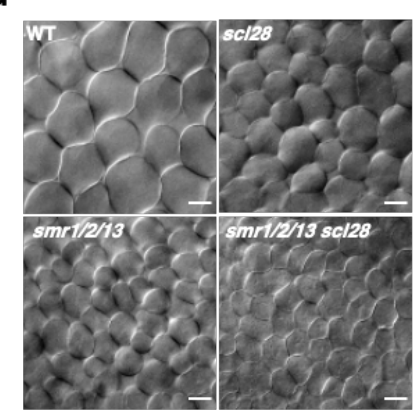

e

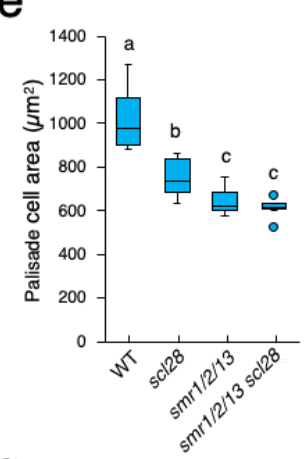

f

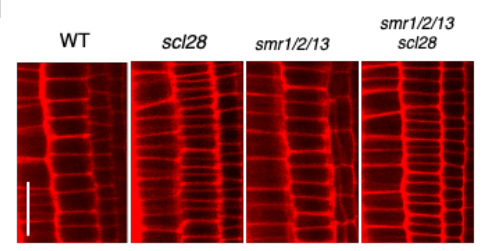

g

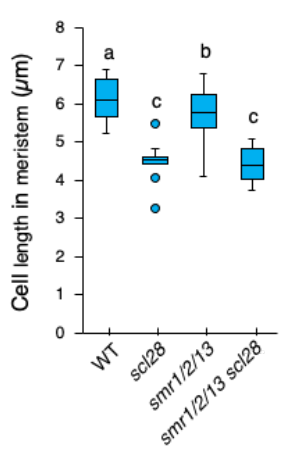

Fig. 7 Cell size control by SCL28-AtSMOS1 is mediated by SMRs.

a Double scl28 atsmos 1 mutation downregulates SMR genes, equivalently to each single mutation. qRTPCR analysis of SMR2, SMR 9 and SMR13 transcripts was performed for comparing expression levels in WT, scl28, atsmosl, and scl28 atsmosl plants. Data are shown as mean \pm SD $(n=3)$. Statistical analysis was performed for each SMR gene based on one-way ANOVA and Tukey's test, and significant differences $(P<0.05)$ were shown in different letters above the bars.

b Upregulation of $S M R$ genes in $S C L 28^{\circ}$ requires presence of AtSMOS1. qRT-PCR analysis was performed for comparing expression levels between atsmos $1, S C L 28^{\mathrm{OE}}$ plants, and those carrying atsmos 1 and $S C L 28^{\circ \mathrm{E}}$ in combination. Two independent lines were used for analyzing SCL2 $8^{\mathrm{OE}} /$ atsmos 1 . Data were statistically analyzed as in (a).

c Co-expression of SCL28 and AtSMOS1 activates the SMR2 promoter in the protoplast transient assay. proSMR2::LUC reporter plasmid was transfected into protoplasts prepared from T87 cells together with expression plasmids of SCL28 and AtSMOS1 as indicated. Schematic presentation of reporter and expression plasmids as shown on the left. Data were statistically analyzed as in (a)

d DIC observations of leaf palisade cells in WT, scl28, smr1/2/13, and smr1/2/13 scl28 plants at 22 DAS.

e Quantification of palisade cell area in the first leaf pairs from plants with indicated genotypes. Quantified data are shown as boxplot (midline $=$ median, box $=\mathrm{IQR}$, whiskers $=1.5 \times \mathrm{IQR}$ ). Different letters above the boxplots indicate significant differences based on one-way ANOVA and Tukey's test. $P<0.05(n=10)$.

f CLSM observation of cortical cell files in root meristems from WT, scl28, smr1/2,/3, and $s m r 1 / 2 / 13$ scl28 plants at 7 DAS. Scale bar indicates $20 \mu \mathrm{m}$. 
g Quantification of cortical cell length in root meristems from plants with indicated genotypes. Quantified data are shown as boxplots as in (e). Different letters above boxplots indicate significant differences as in (e) $(n=10)$. 
a
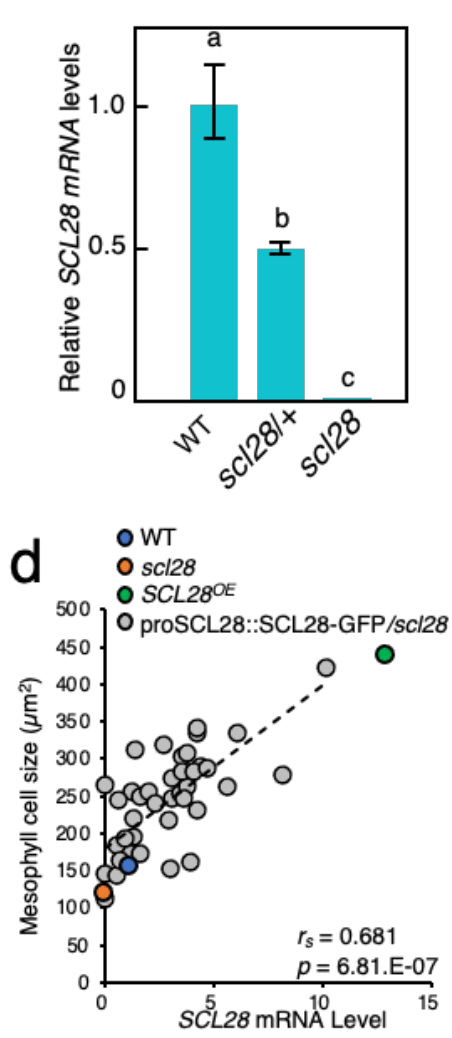

b

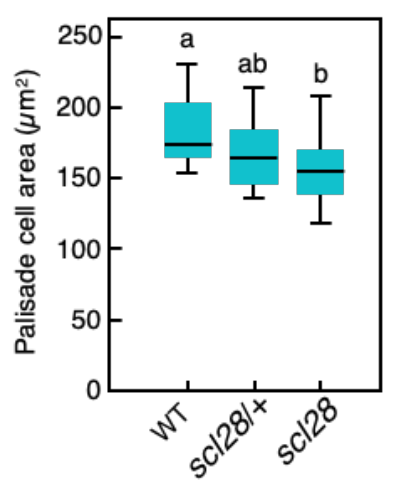

e

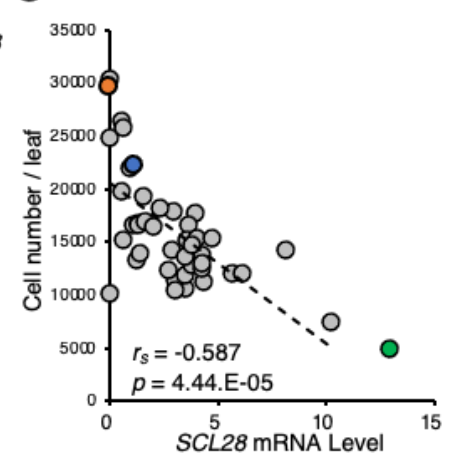

C

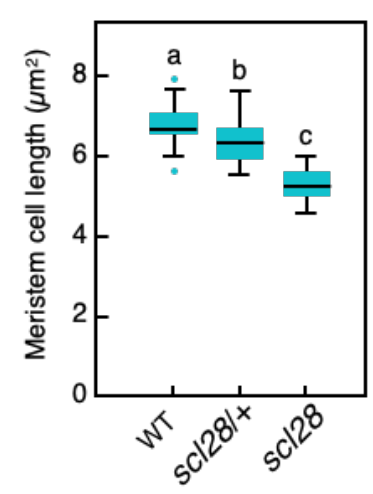

f

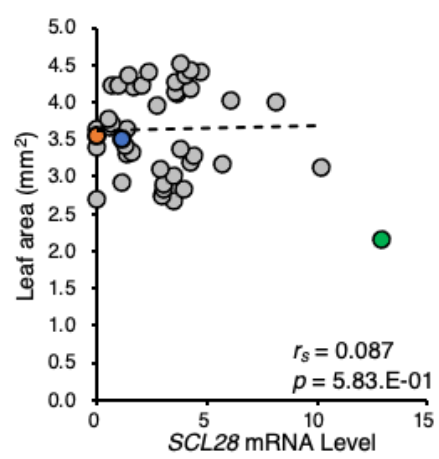

\section{Fig. 8 SCL28 regulates cell size in a dose-dependent manner}

a Moderate downregulation of SCL28 transcripts in plants heterozygous for scl28. qRT-PCR analysis was performed using WT plants, and those heterozygous (scl28/+) and homozygous (scl28) for scl28 at 10 DAS. Data are shown as mean \pm SD $(n=3)$. Statistical analysis was performed based on one-way ANOVA and Tukey's test and significant differences $(P<0.05)$ are shown in different letters above bars. b Quantification of palisade cell area in scl28, scl28/+ and WT plants at 10 DAS. Data are shown as boxplots (midline $=$ median, box $=\mathrm{IQR}$, whiskers $=1.5 \times \mathrm{IQR}$ ), where different letters above boxplots indicate significant differences based on one-way ANOVA and Tukey's test, $P<0.05(n \geq 15)$.

c Quantification of cortical cell length in root meristems of $s c l 28$, scl28/+ and WT plants at 7 DAS. Data are shown as boxplots as in (b). Different letters above the boxplots indicate significant differences as in $(\mathbf{b})(n \geq 12)$.

d Correlation between SCL28 mRNA levels and palisade cell size analyzed by scatterplots, where each dot corresponds to each individual plant from different transgenic lines carrying proSCL28::SCL28GFP under scl28 background. In addition to proSCL28::SCL28-GFP plants (shown in gray dots), WT (blue dot), scl28 (red dot), and SCL28 ${ }^{\mathrm{OE}}$ plants (green dot) were also analyzed.

e Correlation between SCL28 mRNA levels and number of palisade cells per leaf was analyzed by scatterplot as in (d). Symbols are same as in (d).

f Correlation between SCL28 mRNA levels and leaf area was analyzed by scatterplot as in (d). Symbols are same as in $(\mathbf{d})$. 

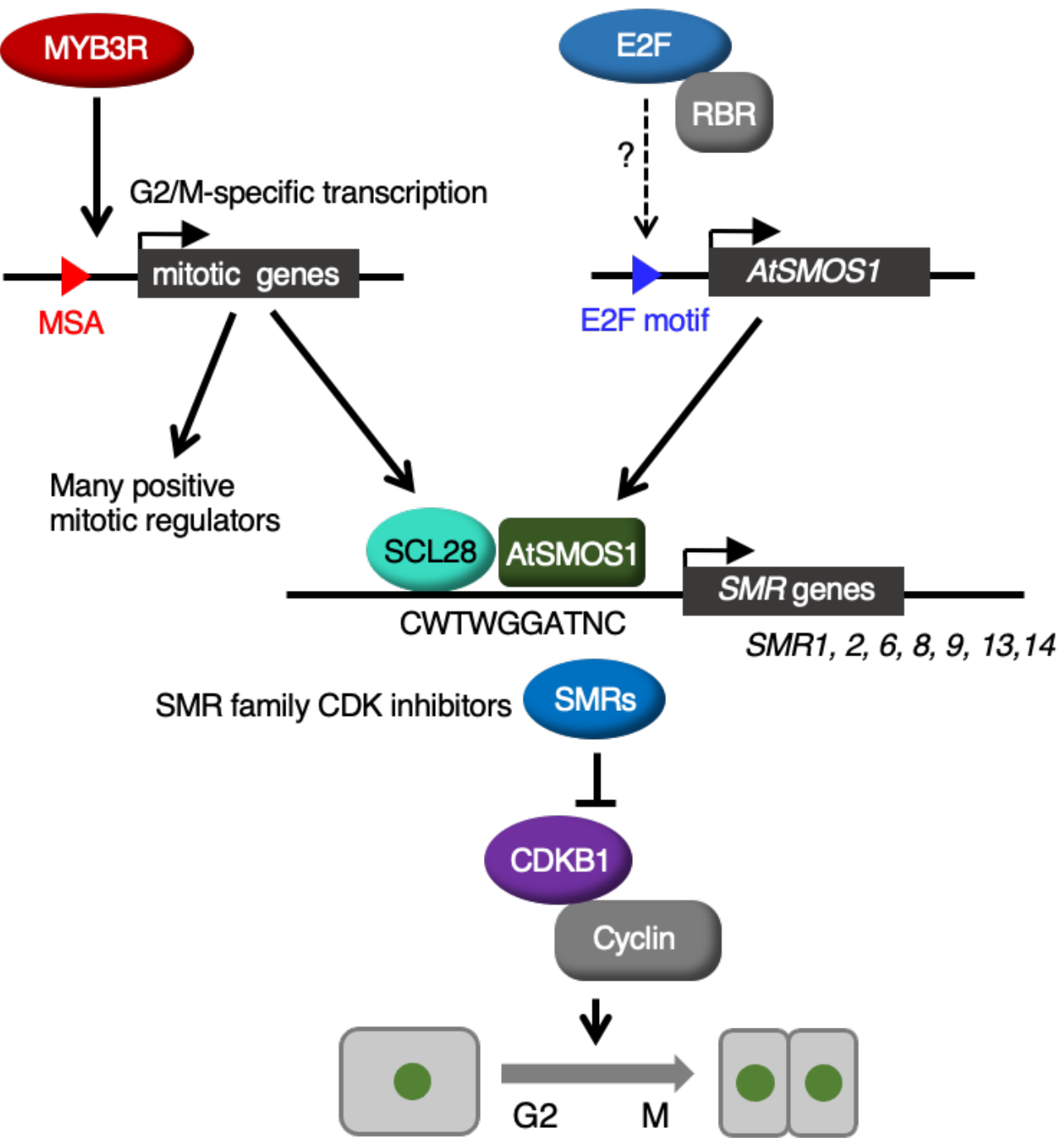

Fig. 9 Schematic model of SCL28-dependent cell size control

MYB3R transcription factors regulate a suite of G2/M-specific genes, including many positive mitotic regulators. However, $S C L 28$, similarly regulated by MYB3Rs acts as a negative regulator of the cell cycle together with AtSMOS1, which may be under the control of the E2F-RBR pathway. Formation of the SCL28-AtSMOS1 heterodimer generates specific binding sequences, enabling direct transcriptional activation of SMR family genes. An increasing amount of SMR proteins, in turn, inhibits CDK activity, thereby negatively influencing cell cycle progression during G2. By controlling the activity of SCL28AtSMOS1 heterodimer, G2 duration in the cell cycle can be finely tuned to maintain a proper balance between cell size and cell number in developing plant organs. 